\title{
Durch Gott legitimiert, das „Reich“ zu befreien
}

\section{Religiöse Semantiken in der Reichsbürgerszene}

\author{
Niklas Herrberg (iD)
}

Eingegangen: 14. Januar 2021 / Überarbeitet: 14. Juni 2021 / Angenommen: 5. Juli 2021 / Online publiziert: 24. Juli 2021

(C) Der/die Autor(en) 2021

Zusammenfassung Der Zusammenhang zwischen der Reichsbürgerszene, religiösen Semantiken und Verschwörungssemantiken wurde trotz soziologischer wie gesellschaftlicher Relevanz für längere Zeit wenig erforscht. Vor dem Hintergrund religiöser Bezüge im deutschen Rechtsextremismus zeigt der Artikel, welche Rolle Religion für die Argumentation der Reichsbürgerszene hat. Dabei wird auf der einen Seite davon ausgegangen, dass Religion und Verschwörungssemantiken als funktionale Äquivalente verstanden werden können, insofern beide deutende, komplexitätsreduzierende und sinnstiftende Funktionen übernehmen. Auf der anderen Seite wird auf inhaltlicher Ebene gezeigt, wie religiöse Semantiken Eingang in die Argumentation der Reichsbürger finden. Die Rolle religiöser Semantiken wird anhand einer qualitativen Inhaltsanalyse online verfügbarer Beiträge von zwölf relevanten Reichsbürgergruppen näher analysiert. Dabei werden Akteurschaftszuschreibungen induktiv erhoben und miteinander verglichen. Der Artikel zeigt, dass die naturalisierende Perspektive der Reichsbürger, die sich in einer Gegenüberstellung von einer als natürlich verstandenen eigenen Akteurschaft und einer als künstlich abgewerteten Akteutschaft zugeschriebener Verschwörer äußert, auch religiös begründet wird: Die eigene Akteurschaft legitimiert sich in diesem Sinne über supernaturalisierende Zuschreibungen. Sie wird als Teileiner göttlich-transzendenten Ordnung begriffen, die in der manichäischen Weltdeutung der Reichsbürger von verschwörerischen Kräften bedroht ist.

Schlüsselwörter Verschwörungstheorien · Reichsbürger · Religiöse Semantiken · Politischer Extremismus · Rechtsextremismus

Niklas Herrberg $(\bowtie)$

Institut für Sozialwissenschaften,Abteilung für Soziologie, Heinrich-Heine-Universität,

Universitätsstr. 1, 40225 Düsseldorf, Deutschland

E-Mail: Niklas.Herrberg@hhu.de 


\title{
Legitimised by God to liberate the "Reich"
}

Religious semantics in the "Reichsbürger-scene"

\begin{abstract}
The connection between the "Reichsbürger"-scene, conspiracy-semantics and religious semantics has been sparsely researched, despite the sociological and societal relevance. In the context of religious references in German right-wing extremism, this article analyses the role of religion for the line of reasoning of the Reichsbürger-scene. On the one hand, religion and conspiracy-semantics can be seen as functional equivalents. Both have the function of interpreting the (social) world, of reducing complexity and of making sense out of experiences. On the other hand, the paper shows how religious semantics are influencing the Reichsbürger's reasoning on the content level. Regarding this relationship, the article presents the results from a qualitative content analysis of online publications from twelve relevant Reichsbürger groups. The analysis aims to inductively specify and to compare the attribution of agency in the line of reasoning. The paper argues that the naturalising perspective of the Reichsbürgers, which is constituted by the attribution of "natural" self-agency and an"artificial" agency of the conspirators, is further justified through religious semantics: In this sense, the self-agency is legitimized by super-naturalising attributions. These attributions are perceived as part of a divinetranscendent order, which is - in the Manichean world interpretation - threatened by conspiratorial forces.
\end{abstract}

Keywords Conspiracy theories · Reichsbürger-scene $\cdot$ Religious semantics · Political extremism $\cdot$ Right-wing extremism

\section{Einführung}

„Aber nein, wir sind nicht frei. Wir sind immer noch ein besetztes Land. Deutschland hat keinen Friedensvertrag und dementsprechend ist Deutschland auch kein echtes Land" sagte der Sänger Xavier Naidoo im ZDF-Morgenmagazin bereits am 25.05.2011 ${ }^{1}$. Die These, dass die heutige Bundesrepublik kein souveräner Staat und das deutsche Volk folglich unfrei sei, kann als Kernbestandteil der Überzeugung der sogenannten „Reichsbürger“ verstanden werden. Xavier Naidoo ist in den letzten Jahren immer wieder als einer der prominentesten Befürworter derartiger Verschwörungssemantiken in Erscheinung getreten. Neben dem Glauben an eine groß angelegte Verschwörung gegen ,die“ Deutschen ist der Sänger ferner auch für seine starke Bezugnahme auf christliche Motive und Symboliken bekannt, die er sowohl in seinen Liedern als auch in öffentlichen Äußerungen aufgreift. Gerade die Überzeugung, im religiösen Sinne in einer Endzeit zu leben, sticht hier hervor (vgl. Pilz 2014; Schwarzer 2020). In gewisser Weise demonstriert Xavier Naidoo so prägnant eine Verbindung zwischen religiösen Endzeit-Motiven und reichsbürgerlichem Verschwörungsglauben, die weit über seine Person hinausreicht. An diesen Umstand anknüpfend untersuche ich in diesem Beitrag, wie in der Reichsbürgersze-

\footnotetext{
1 Ein Videoausschnitt kann über einen Link im Artikel von Schwarzer (2020) abgerufen werden.
} 
ne der Glaube an eine Verschwörung gegen Deutschland in einem Zusammenhang mit unterschiedlichen religiösen Motiven steht. Hierbei steht weniger eine Analyse religiöser Motive einzelner Reichsbürger als vielmehr eine Betrachtung derartiger Inhalte auf Ebene verschiedener Reichsbürgergruppen im Vordergrund.

Obwohl die Entstehung der Reichbürgerszene bis (mindestens) in die 1980erJahre zurückverfolgt werden kann (vgl. Rathje 2017a), wird ihr erst in den letzten fünf bis sechs Jahren in der Forschung wie auch in der breiteren Öffentlichkeit Aufmerksamkeit geschenkt. Einfluss auf diesen Wandel der wissenschaftlichen und öffentlichen Wahrnehmung hatten beispielsweise der Mord an einem Polizeibeamten durch einen Reichsbürger im Oktober 2016 (vgl. Jansen und Fröhlich 2016) oder die Teilnahme vieler Reichsbürger an den Protesten gegen die Corona-Schutzmaßnahmen seit Frühling 2020. Eine tiefergehende Erforschung der Reichsbürgerszene steht jedoch noch an ihrem Anfang. Dementsprechend ist es sinnvoll, sich vor allem auch mit den in der Reichsbürgerszene vorherrschenden Überzeugungen auseinanderzusetzen, da von ihnen ausgehend die Spezifik der Szene genauer herausgearbeitet werden kann.

Zweierlei ist in diesem Zusammenhang zu berücksichtigen. Zum einen hat sich in der Forschung gezeigt, dass die Reichsbürgerszene nicht als kohärente Bewegung angesehen werden kann, sondern aus divergierenden und heterogenen Gruppen sowie Einzelpersonen besteht, für die unterschiedliche Überzeugungen im Vordergrund stehen (vgl. Rathje 2017a; Keil 2018; Speit 2017). Zum anderen möchte ich aufzeigen, dass gleichwohl zentrale Motive und Argumentationen auffindbar sind, die von verschiedenen Gruppen geteilt werden. Wie im Folgenden anhand einer explorativen Inhaltsanalyse gezeigt wird, sind dies insbesondere: (1) eine Zentrierung auf Deutschland, (2) antisemitische Stereotype, (3) der Bezug auf eine vermeintliche Entrechtung und (4) Manipulation der Deutschen sowie (5) der Aufruf zum Freiheitskampf gegen die Bundesrepublik. Diese Themenfelder wurden auf Basis von In-Vivo-Codes in einem inhaltsvergleichenden Typisierungsprozesse entwickelt, da so bisher wenig untersuchte Argumentationszusammenhänge erhoben und analysiert werden konnten. Durch diesen Typisierungsprozess ist es des Weiteren möglich zu zeigen, in welcher Weise Religion in diesem Zusammenhang als relevanter Aspekt der Argumentation anzusehen ist ${ }^{2}$.

Die Argumentationen der Reichsbürger können soziologisch wiederum als $\mathrm{Zu}$ schreibung von Akteurschaft verstanden werden, die klar zwischen einer eigenen Akteurschaft als Reichsbürger und der Akteurschaft vermeintlicher Verschwörer unterscheidet. Durch diese (personalisierenden) Zuschreibungen ist es den Reichsbürgern möglich, gesellschaftliche Prozesse und Ereignisse zu deuten und einen Umgang damit zu entwickeln (vgl. Barkun 2003; Cubitt 1989). Indem sie die Welt durch das Handeln (über-)mächtiger Akteure erklären, weisen die reichsbürgerlichen Argumentationen zum einen funktionale Analogien zu (fundamentalistischen) religiösen Überzeugungen auf, sodass ich religiösen Glauben und den Glauben an wirk-

\footnotetext{
2 Hierbei ist zu betonen, dass nicht spezifisch nach religiösen Inhalten erhoben oder analysiert wurde. Durch die explorative Ausrichtung der Inhaltsanalyse konnte die Relevanz von Religion induktiv aus dem analysierten Material bestimmt werden, da in unterschiedlichen Argumentationszusammenhängen auf religiöse Semantiken zurückgegriffen wurde.
} 
mächtige Verschwörungen in einigen Aspekten als funktionale Äquivalente ansehe (vgl. Krüger und Seiffert-Brockmann 2018). Zum anderen möchte ich auf inhaltlicher Ebene nachvollziehen, wie die Verschwörungssemantiken der Reichsbürger Elemente und Motive verschiedener religiöser Semantiken aufnehmen, in ihrem Sinne interpretieren und mit ihnen eigene Handlungen legitimieren. Unter Semantik ist mit Holz allgemein ein ,in sich strukturiertes Syndrom von Sinngehalten“ (Holz 2010, S. 28) zu verstehen, das in Anknüpfung an Luhmann einen (Re-)Produktionsprozess von Sinn bezeichnet, der sich durch eine höherstufige Generalisierbarkeit und relative Situationsunabhängigkeit auszeichnet (vgl. Holz 2010).

Der Beitrag ist folgendermaßen aufgebaut: In Kapitel zwei und drei skizziere ich knapp bisherige Forschungsarbeiten zur Reichsbürgerszene sowie zur allgemeinen Thematisierung von Religion im Rechtsextremismus, wobei diese Aspekte den Kontext der weiteren Untersuchung abstecken. In den beiden daran anknüpfenden Kapiteln erläutere ich in zweifacher Hinsicht den Zusammenhang zwischen Religion und der Reichsbürgerszene: Im vierten Kapitel führe ich aus, in welcher Weise der Verschwörungsglaube unter Reichsbürgern mit Religion als funktionalem Äquivalent verbunden ist. Das fünfte Kapitel wiederum widmet sich der Frage, wie auf thematisch-substantieller Ebene religiöse Semantiken Eingang in reichsbürgerliche Argumentationen finden. Bei diesem Aspekt beziehe ich mich zentral auf die Ergebnisse einer explorativ ausgerichteten qualitativen Inhaltsanalyse, mit der digitale Veröffentlichungen von zwölf relevanten Reichsbürgergruppen ausgewertet wurden. Auch wenn dabei der Fokus auf einer Exploration der sich allgemein zeigenden Akteurschaftszuschreibungen lag, zeigte sich im Fortgang der induktiven Analysestrategie, dass religiöse Semantiken in allen identifizierten Kernthemen gefunden werden können und dort jeweils als wichtige Legitimation der Argumentation in Erscheinung treten.

\section{Die Reichsbürgerszene}

Bei einer Einordnung der Reichsbürgerszene kommt man nicht umhin, sie im Kontext des Rechtsextremismus in Deutschland zu bestimmen. Unter Rechtsextremismus ist hier eine durchaus ,bewegungsmäßig organisierte Erscheinung“ (Quent 2015, S. 10) zu verstehen, die sich aus unterschiedlichen Akteuren zusammensetzt. In Anknüpfung an Quent (2015) kann festgehalten werden, dass trotz Heterogenität der ,praktizierte, manifeste Rechtsextremismus (...) verschiedene Handlungs- und Organisationsformen mit der ideologischen Basis sozialer Ungleichwertigkeitsvorstellungen beschreibt“, wobei „Rechtsextreme (...) auf die alleinige, rassistisch beziehungsweise nationalistisch begründete Vormachtstellung innerhalb bestimmbarer sozialer, kultureller, politischer oder räumlicher Bereiche (zielen)“" (Quent 2015, S. 10). Ferner ist für die Betrachtung der Reichsbürgerszene im Kontext des Rechtsextremismus besonders das Konzept der Reichsideologie relevant (vgl. Stöss 2010). Laut Richard Stöss (2010) zeichne sich der deutsche Rechtsextremismus nach 1945 thematisch unter anderem durch zwei Momente aus: erstens den deutschen Staat als nicht legitim und/oder legal anzusehen und zweitens von einer Fortexistenz des Deutschen Reichs auszugehen. Daran anknüpfend hat Jan Rathje aufgezeigt, dass 
auch die Reichsbürgerszene von dieser Idee zusammengehalten werde (vgl. Rathje 2017a; Begrich und Speit 2017). Er geht über Stöss hinaus, indem er anhand unterschiedlicher Kombinationen der Aspekte der Reichsideologie verschiedene Typen bestimmt: Sowohl (1) klassische Rechtsextreme seit 1945 als auch (2) Reichsbürger im eigentliche Sinne artikulieren demnach beide Aspekte der Reichsideologie. Die Unterscheidung zwischen diesen beiden Gruppen stellt sich für Rathje aus einer unterschiedlichen Betonung der Aspekte her: Die Kategorie der Rechtsextremen seit 1945 bilde die historischen Ursprünge der Reichsideologie ab. In ihr würden rechtsextreme Gruppen und Einzelpersonen gefasst, die, wie Stöss (2010) darlegt, seit 1945 an der Kampagne zur Wiederherstellung des Deutschen Reiches in den Grenzen von 1937 beziehungsweise 1939 beteiligt waren und sind. Die Kategorie der ,Reichsbürger“ beinhalte wiederum diejenigen, die in der Traditionslinie von Wolfgang Ebel - dem Gründer der ,kommissarischen Reichsregierung“, die als Prototyp heutiger „Regierungen“ der Reichsbürger gelten kann - bereits in einem Deutschen Reich zu leben glauben und deren Identitätskonstruktionen und Handlungen maßgeblich von ihrer Reichsbürgerschaft bestimmt würden (vgl. Rathje 2017a). (3) „Selbstverwalter“ gehen dagegen der Typologie zufolge von der Nichtlegitimität und -legalität der Bundesrepublik aus, ohne zugleich die Fortexistenz des Deutschen Reichs zu postulieren. Abschließend umfasse hier (4) die Gruppe der „Souveränitätsfordernden“3 Personen, die nur von einer Illegitimität ausgehen, aber nicht von einer Illegalität der Bundesrepublik überzeugt sind. Alle Typen sind für Rathje durch die Verschwörungssemantik verbunden und eingefasst, nach der die Bundesrepublik von hintergründigen und fremden Mächten beherrscht werde (vgl. Rathje 2017a).

Trotz der hier ersichtlichen Zusammenhänge zwischen klassischem Rechtsextremismus und Reichsbürgern wurden letztere bis vor einigen Jahren eher als Kuriosität abgetan, die zwar Phantasiestaaten, -reiche und -königreiche gründen, die eigene Wohnung oder das eigene Grundstück zum Kleinstaat deklarieren, Informationen über eine vermeintliche Besatzung Deutschlands zu Verfügung stellen oder als Querulanten Verwaltungseinrichtungen und staatlichen Behörden Ärger bereiten, aber kaum eine ernste Bedrohung für eine aufgeklärte und offene Gesellschaft darstellen (vgl. Rathje 2017a; Keil 2018). Wie einleitend angeführt, fand die Szene - zu der nach Angaben des Bundesinnenministeriums immerhin gut 19.000 Personen gezählt werden können - erst in den letzten Jahren verstärkt Eingang in eine breitere Medienöffentlichkeit, wobei rückblickend selbst staatliche Behörden eingestehen mussten, dieser Szene nicht genügend Aufmerksamkeit entgegengebracht zu haben ${ }^{4}$ (vgl. Bundesministerium des Innern, für Bau und Heimat 2019; Zeit Online 2016a; Zeit Online 2016b).

Dieses aufkommende Interesse schlug sich auch wissenschaftlich nieder, sodass derzeit einige erste Untersuchungen zu verschiedenen Aspekten des Phänomens vorliegen. So wurde neben einer eher anwendungsbezogenen Perspektive, die primär pädagogische und juristische Aspekte sowie Aspekte der politischen Bildung

\footnotetext{
3 Bei diesem Typ ist ferner auch eine deutliche Verbindung zur sog. „,neuen Rechten“ ersichtlich.

4 Auch eine intensivere Beschäftigung der Sicherheitsbehörden mit Reichsbürgern ist in diesem Zusammenhang erst seit Mitte der 2010er Jahre zu beobachten.
} 
thematisiert ${ }^{5}$, auch in inhaltlich-organisatorischer Hinsicht die Reichsbürgerszene bereits behandelt. Dies geschah einerseits - wie oben in Bezug auf Rathjes Typologie geschehen - durch allgemeine Betrachtungen und Einordnungen; andererseits finden sich auch Studien zu spezifischen (inhaltlichen) Aspekten der Szene. So konnte beispielweise herausgearbeitet werden, dass Antisemitismus ein ,zentrales Strukturelement der Ideologie des Reichsbürger-Milieus“ (Rathje 2017b) darstellt. Auch in Hinblick auf Geschlechterbilder und -rollen konnten inhaltliche Spezifika ausgemacht werden, die für Bischof aus einer „Krise der Männlichkeit“ (Bischof 2017) resultieren und eine Rückorientierung an einem „,klassischen“ Männlichkeitsbild implizieren. Ferner möchte ich auch schon an dieser Stelle auf esoterische Konzepte verweisen, die sich exemplarisch im Gebrauch der ,neuen germanischen Medizin“ manifestieren. Darunter ist eine „Alternative“ zu gängigen Behandlungspraxen zu verstehen, die sich dadurch ,auszeichnet“, dass sie somatische Leiden prinzipiell durch psychische Konflikte erklärt (vgl. Baeck 2017; Hardinghaus 2016).

Während Antisemitismus zwar in Form eines christlich geprägten Antijudaismus durchaus eine explizit religiöse Dimension aufweisen kann, ist der Zusammenhang zwischen religiösen Semantiken und der Reichsbürgerszene gerade auch bei der Thematisierung von Esoterik deutlich. Nichtsdestotrotz bedeutet diese einzelthematische Darstellung nicht, dass religiöse Aspekte bisher systematisch mit Argumentationsmustern der Reichsbürger in Bezug gesetzt wurden. Es ist eher zu konstatieren, dass diese Muster in Bezug auf den Gegenstand der Reichsbürger oftmals unvermittelt nebeneinanderstehen und nur punktuell aufeinander bezogen wurden.

\section{Religiöse Inhalte im Rechtsextremismus}

Die Bezugnahme auf Religion innerhalb des Rechtsextremismus kann durchaus als vielfältig beschrieben werden und soll hier nicht erschöpfend behandelt werden. Vielmehr geht es an dieser Stelle um eine Skizze der Beziehung, vor deren Hintergrund ich die Argumentationen innerhalb der Reichsbürgerszene betrachten werde. In diesem Zusammenhang unterscheide ich im Folgendem grob zwischen einer eher impliziten und einer eher expliziten Thematisierung von religiösen Semantiken. Unter einer impliziten Thematisierung verstehe ich hier, dass religiöse Inhalte in nicht unmittelbar offensichtlicher Weise kommuniziert werden, also Begriffe, die klar mit institutionalisierten Religionsgemeinschaften in Verbindung stehen, nicht angesprochen werden. Vielmehr kommt es bei einer impliziten Thematisierung dazu, dass eigene Begriffe rechtsextremer Akteure eine religiöse Konnotation erhalten - eine explizite Thematisierung greift demgegenüber konkret mit Religionsgemeinschaften verbindbare und von ihnen verwendete Begriff auf.

\footnotetext{
5 Zum einen werden hier juristische Aspekte angesprochen, die sich gerade um die Fragen nach Waffenbesitz von Reichsbürgern und der Verweigerung, Steuern zu zahlen, drehen. Zum anderen wird verschiedentlich auch der Handlungsspielraum kommunaler Behörden thematisiert sowie darüber hinaus danach geforscht, inwieweit Bildungsangebote und Prävention den Gefahren der Reichsbürgerszene entgegenwirken können (vgl. Vormbaum 2017; Müller und Rebler 2019; Adensamer 2017; Caspar und Neubauer 2015; Keil 2018; Haase 2018).
} 
In impliziter Hinsicht kann zentral auf das Motiv der (nationalen) Erlösung der eigenen Gruppe verwiesen werden, das sich religiöser Topoi als Form einer säkularisierten Religion (beziehungsweise einer ,diesseitigen Religion“) bedient (vgl. Hoyningen-Huene 2003). Daran schließt sich an, dass es bei rechtsextremen Akteuren zu einer Anknüpfung an Vorstellungen des Vorhandenseins transzendenter Mächte kommen kann, die die Geschicke der Welt und/oder Völker maßgeblich prägen. Dies kann sich sowohl in Form von Mystifizierungen des eigenen Volkes und der eigenen Nation als auch einer, ,gegebenen“ Ordnung als solcher äußern (vgl. Heller 2002). Der Verweis auf derartige Kräfte schlägt eine wichtige Brücke zu Narrativen moderner Esoterik, die sich zwar weniger deutlich auf nationale und völkisch konzipierte Kollektive beziehen, jedoch in ähnlicher Weise eine transzendente Konstitution der Welt annehmen und teilweise zu einer imaginierten Ursprünglichkeit zurückkehren wollen, die in ähnlicher Form auch im Rechtsextremismus Thema ist (vgl. auch Buschbom 2017).

Die Anknüpfung an die heterogenen Vorstellungen und Praktiken der modernen Esoterik deuten dabei wiederum schon in Richtung der expliziten Thematisierung religiöser Begrifflichkeiten. Stärker als in Bezug auf implizite Anknüpfungen zeigen sich hier Widersprüche und konkurrierende Vorstellungen. Dies betrifft zentral die Frage, auf welche religiöse ,Traditionslinie“ Bezug genommen wird. Gerade im deutschen Kontext sind hier zwei wichtige Argumentationen zu finden. In den eindeutig und offen am Nationalsozialismus orientierten Teilen des Rechtsextremismus findet vor allem ein Bezug zur als ,ursprünglich germanisch“ imaginierten nordischen Mythologie statt, die als ,wahre“ Religion vorgestellt wird - obgleich es sich hierbei eher um eine projektive Bezugnahme zur Konstruktion einer ,traditionellen“ germanisch-religiösen Identität handelt, die heutige Weltreligionen als Teil einer negativ konnotierten Moderne ablehnt ${ }^{6}$ (vgl. Cantzen 2015; Kellershohn 1998). Demgegenüber kann gleichzeitig beobachtet werden, dass sich andere Teile des Rechtsextremismus eher in eine ,christlich-abendländische“ Tradition stellen. Diese Tradition ist nicht zuletzt dadurch gekennzeichnet, dass sie von einer ,islamischorientalischen“ Kultur abgegrenzt wird, die die eigene Kultur bedrohen würde ${ }^{8}$. Wie Salzborn (2015) und auf ähnliche Art Weiß (2017) überzeugend darlegen, fungiere der Islam hierbei primär als ein Projekt der (kulturellen) Identitätsstiftung, mit dem die eigene Kultur in einem Konkurrenzverhältnis stehe. $\mathrm{Zu}$ dieser Dichotomisierung komme hinzu, dass insbesondere dem Judentum als einem „Tertium non datur" (Holz 2010) weiteres Gefahrenpotential zugeschrieben werde, das nicht nur eine konkurrierende Identität repräsentiere, sondern vermeintlich Identität als solche unterminiere.

\footnotetext{
${ }^{6}$ Eine solche Ablehnung kann nicht zuletzt antisemitische Züge aufweisen.

7 Trotz der differenten Bezugnahme stimmen beide „Traditionslinien“ wiederum darin überein, dass sie Religion im Kontext einer kulturellen Identitätsstiftung verorten und sie somit als wichtiges Element einer heute zu aktualisierenden Form kultureller Praxis und Identität verstehen (vgl. Bischoff und Arnold 2017; Wiedemann 2016).

8 Anzumerken ist, dass diese Traditionslinien hier eher als idealtypische Heuristiken verstanden werden sollten, bei denen es durchaus zu (widersprüchlichen) Überschneidungen kommen kann.
} 


\section{Religiöse Semantiken als funktionale Äquivalente zum Verschwörungsglauben der Reichsbürger}

Wie einleitend angeführt, begreife ich die Argumentationslogik der Reichsbürgerszene als „Verschwörungssemantik“, die durch einen Glauben an eine Verschwörung gegen Deutschland und das Deutsche Reich konstituiert wird. Dieser Glauben kann hierbei funktionalistisch mit religiösen Semantiken in Beziehung gesetzt werden. Bevor ich im Weiteren diesen Zusammenhang näher beleuchte, möchte ich den Begriff der Verschwörungssemantik genauer bestimmen.

Verschwörungssemantiken können als Weltdeutungssysteme verstanden werden. In diesem Sinne stellen sie für Andreas Anton als „heterodoxe Wissensordnung“9 einen Zugang zur Welt dar, der als ,Überzeugungssystem oder Erklärungs- beziehungsweise Deutungsmodell (...) aktuelle oder historische Ereignisse, kollektive Erfahrungen oder die Entwicklung einer Gesellschaft insgesamt als die Folge einer Verschwörung interpretiert“ (Anton 2014, S. 117). Mit dieser Definition des Phänomens tritt eine entscheidende Dimension in den Vordergrund: Für Verschwörungssemantiken ist es relevant, dass eine bestimmbare Gruppe - die (zumeist im Geheimen bzw. verborgen arbeitenden) Verschwörer - als kausal verursachend für soziale Prozesse angesehen wird. Bei dieser Determination der sozialen Welt ist es darüber hinaus relevant, dass die Verschwörer als intentional handelnde Gruppe begriffen werden, die besagte historische Ereignisse, Erfahrungen und Entwicklungen bewusst planend herbeiführen würden (vgl. Barkun 2003; Cubitt 1989; Harder 2018). Im Rahmen der Reichsbürgerszene ist dies die Vorstellung, dass geheime und verschwörerisch agierende Mächte ,die Deutschen“ mittels der als illegitim und illegal verstandenen Bundesrepublik lenken beziehungsweise durch die Bundesrepublik ihre verborgenen Ziele verfolgen würden.

Intentional handelnde Akteure stellen spätestens seit Webers Anspruch an eine verstehende Soziologie, die soziale Phänomene ,deutend verstehen und (...) ursächlich erklären“ (Weber 2002, S. 1) soll, eine relevante soziologische Kategorie dar. Insofern in Anknüpfung an dieses Verständnis die soziologische Theoriebildung die Frage nach intentionaler Handlung zu einer ihrer wichtigsten Begriffskomplexe ausbaute, können auch Verschwörungssemantiken unter Berücksichtigung dieser Kernelemente der Soziologie begriffen werden (vgl. Kron und Winter 2009; Helfferich 2012). Betrachtet man aus dieser Perspektive die Frage nach intentionaler Akteurschaft, ist es wichtig zu betonen, dass in sozialen Interaktionen Intentionalität immer ein Moment praktischer Konstruktion beinhaltet: Beim wechselseitigen Bezug aufeinander können Akteure nur von ,außen“ auf die jeweilige Intentionalität des Anderen schließen. Intentionale Akteurschaft basiert somit schon auf Ebene alltäglicher Interaktion teilweise auf Zuschreibung (vgl. Helfferich 2012; Greve 2012).

Verschwörungssemantiken müssen jedoch jenseits solcher interdependenten Situationen als eine Form der Zuschreibung von Akteurschaft aufgefasst werden, da von einer „einseitigen“ Konstruktion gesprochen werden kann. Diejenigen, die

\footnotetext{
9 Mit dem Begriff der „Heterodoxität“ macht Anton kenntlich, dass Verschwörungssemantiken oftmals - und so auch im Falle der Reichsbürger - von den „orthodoxen“ Weltdeutungs- und Überzeugungssystemen (mehrheits-)gesellschaftlich legitim anerkannter Deutungsinstanzen abweichen.
} 
an eine Verschwörung glauben, schreiben Akteurschaft auf eine subjektiv wahrgenommene Verschwörung zu. Dies ist insofern nicht als interdependent zu begreifen, als dass die zugeschriebenen Verschwörer ihrerseits in der Regel nicht „zurückzuschreiben“"10. Durch diese Situation ist es möglich, dass Verschwörungssemantiken sich von der Wahrnehmung realer Akteurschaft ,entkoppeln“. Zugleich fällt so die wechselseitige Anerkennung zwischen interdependenten Zuschreibungen weg, die die Geltungen von Zuschreibungen regulieren kann: Akteurschaftszuschreibungen können somit als potenziell von realen Vorgängen im Sozialen verselbstständigte Phänomene existieren. Dabei muss in Rechnung gestellt werden, dass solche Akteurschaftszuschreibungen, auch wenn sie nicht an reale Akteurskonstellationen anknüpfen oder diese grob verzerrt wahrnehmen, dennoch soziale Realität prägen können. Indem eigenes Handeln an subjektiv wahrgenommenen Verschwörungen ausgerichtet wird, können derartige Semantiken wirksame Deutungsmuster erzeugen und Handlungsdynamiken anstoßen, die Einfluss auf gesellschaftliche Prozesse nehmen (vgl. Anton 2011; Butter 2018).

In den vorherigen Absätzen habe ich vor allem die allgemeine Logik von Verschwörungssemantiken verdeutlicht. Es ist jedoch offen geblieben, warum es zu solchen Deutungen kommt und in welcher Weise sie mit Religion verknüpft sind. Um diesen Zusammenhang adäquat zu thematisieren, ist es notwendig, auf das Verhältnis von Verschwörungssemantik und Religion einerseits sowie auf die allgemeine Entwicklung (spät-)moderner Vergesellschaftung andererseits zu fokussieren: „Während das rationale Weltbild das christlich-mythische ablöste, erlaubten sie es, ,das etablierte heilsgeschichtliche Strukturmuster, demzufolge alles, was geschieht, so geplant worden ist, zu säkularisieren“ (Butter 2014, S. 261). Mit anderen Worten: Die Verschwörer nehmen den Platz Gottes als Weltenlenker ein, und ein mechanistisches Weltbild von Ursache und Wirkung kann so auch im Angesicht von Chaos und Kontingenz in dezentralisierten, funktional differenzierten modernen Gesellschaften aufrechterhalten werden. Verschwörungstheorie also auch als (Abwehr-)Reaktion auf Modernisierung und zunehmende Komplexität, als Ausdruck der Sehnsucht nach Einfachheit, Determiniertheit und Übersichtlichkeit - die mit ihrem radikalen Misstrauen allerdings auch in der kulturellen Logik der Moderne liegt" (Krüger und Seiffert-Brockmann 2018, S. 72).

In diesem Verständnis erscheinen Verschwörungssemantiken strukturell als „Erben" der Welterklärungsfunktion von Religion. Doch auch jenseits dieses zeitlichen Nacheinanders erscheinen Verschwörungssemantiken und Religion als einander ähnliche „Alternativsemantiken“ zu einem soziologischen Bild der (Spät-)Moderne. In diesem Bild wird die (Spät-)Moderne als Vergesellschaftungsform charakterisiert, die einerseits sowohl von abstrakten Zusammenhängen, widersprüchlichen Dynamiken und systemischen Sachzwängen als auch andererseits von kontingenten und

$10 \mathrm{Zu}$ beachten ist hierbei, dass verschwörerische Gruppen teilweise auch gänzlich als Zuschreibungen aufgefasst werden müssen. 
ungeplanten Prozessen gekennzeichnet ist ${ }^{11}$. Verschwörungssemantiken und Religion ist demgegenüber zu eigen, eine Erzählung zu etablieren, die ambivalente Uneindeutigkeiten minimiert und klare Zurechenbarkeit attribuiert.

Über diese epistemische Ebene der Komplexitätsreduktion hinausgehend beschränkt sich die funktionale Ähnlichkeit hinsichtlich der Orientierung in der (sozialen) Welt nicht nur auf eindeutige Erklärungsangebote. Auch hinsichtlich der Möglichkeit von Sinnstiftung können Religion - vor allem in ihrer fundamentalistischen Ausdeutung - und Verschwörungssemantik als funktionale Äquivalente angesehen werden. So sind beide in vielen Fällen durch einen deutlichen Manichäismus gekennzeichnet (vgl. Barkun 2003; Cubitt 1989): Die positiv charakterisierte In-Group der (Verschwörungs-)Gläubigen sieht sich einer Gefahr durch das Agieren finsterer Mächte gegenüber, die durch spezifische (und teilweise ritualisierte) Handlungspraxen ,abgewehrt“ werden können. In diesem Kontext tritt hervor, dass beide Phänomene auch in Bezug auf ihre Rolle für die Entwicklung und Stabilisierung von sozialen/kollektiven Identitäten vergleichbar sind. Insofern können beide auch in praktischer Hinsicht handlungsanleitend fungieren: Beide erzeugen auf gemeinsamen Überzeugungen basierende Kollektive, die dem Einzelnen Normvorstellungen an die Hand geben, sodass Handlungen (wertrational) daran orientiert werden können. Verschwörungssemantiken und fundamentalistische Religionsverständnisse erscheinen so in vielfältiger Weise als funktionale Äquivalente im Umgang mit den Anforderungen moderner Vergesellschaftungsformen (vgl. Wössner 1973; Douglas et al. 2019).

In den letzten Absätzen bin ich vor allem von einer strukturlogischen Vergleichbarkeit von Verschwörungssemantiken und Religion ausgegangen. Die enge funktionalistische Verzahnung ergibt jedoch im Weiteren ein Problem: Beide sind nicht die einzigen Deutungssysteme, die Komplexität reduzieren und Orientierung stiften können. Beispielsweise können auch andere politische Ideologien ${ }^{12}$, (sub-)kulturelle Überzeugungen, Traditionen oder der Glaube an den ,gesunden Menschenverstand“ als weitere funktionale Äquivalente bestimmt werden. Kurz gesagt: Der Vergleich verbleibt zu stark auf Ebene der Form und berücksichtigt nicht die Spezifik einer inhaltlichen Dimension. Vor dem Hintergrund dieses Argumentes erscheint es mir geboten, genauer auf die religiösen Semantiken in den Argumentationen der Reichsbürgerszene einzugehen. Als religiöse Semantiken begreife ich (in Bezug auf die inhaltliche Ebene) in einem sehr weiten Verständnis verschiedene Formen strukturierter Sinn(re-)produktionen (vgl. Holz 2010), die von ,klassischen“ Religionsgemeinschaften, neuen religiösen Bewegungen oder individualisierten und uninstitutionalisierten Religions- und Glaubenspraxen geäußert werden. Dies bedeutet, dass

\footnotetext{
11 Paradigmatisch kann hier auf so unterschiedliche Theoretiker wie Karl Marx, Max Weber, Émile Durkheim oder Niklas Luhmann verwiesen werden. Trotz deutlicher Differenzen in ihren jeweiligen Zugängen zeigen sich dahingehend Parallelen, dass moderne Gesellschaften durch apersonale, sachlich-bürokratische und verselbstständigte Beziehungen zwischen Menschen gekennzeichnet werden, sodass „die“ Moderne als komplexes und interdependentes Gesamtgefüge menschlicher Praxen und Kommunikationen erscheint (vgl. Marx 2009; Weber 2002; Durkheim 1999; Luhmann 2005).

12 Die Charakterisierung der Reichsbürgerszene durch rechtsextreme Verschwörungssemantiken kann als eine spezifische Form der politischen Ideologie verstanden werden, die den Stellenwert des Glaubens an eine (umfassende) Verschwörung als zentrales Element des Überzeugungssystems bestimmt.
} 
ich mich im Folgendem näher mit den konkreten Inhalten aus der Reichsbürgerszene auseinandersetzen werde, die an derartige Semantiken anknüpfen. Hierbei werde ich zentral auf Semantiken eingehen, die sich explizit auf transzendente Wesenheiten, Prinzipien und/oder Mächte beziehen. Das Ziel ist, deren Rolle und Einbettung in übergreifende Argumentationen der Reichsbürger nachzuvollziehen.

\section{Inhaltsanalytische Untersuchung religiöser Semantiken bei Reichsbürgern}

Zur Analyse der Semantiken stütze ich mich im Folgendem auf die Resultate einer qualitativen Inhaltsanalyse, bei der die Beiträge auf den Webseiten von zwölf Reichsbürgergruppen ausgewertet wurden. Insgesamt wurden dabei 1046 Beiträge erhoben und analysiert. Die Auswahl hat den Anspruch, möglichst relevante Reichsbürgergruppen abzubilden, von denen wiederum alle verfügbaren Beiträge in die Inhaltsanalyse eingegangen sind..$^{13}$

Ich knüpfe dabei mit der Analyse an das Verständnis der reichsbürgerlichen Verschwörungssemantik als Zuschreibung von Akteurschaft an. Es ist mein Ziel, verschiedene Akteurschaftszuschreibungen als In-Vivo-Codes zu erheben und durch eine vergleichende Typologisierung zu übergeordneten Themenfeldern zu systematisieren (vgl. Kelle und Kluge 2010). Hierzu unterscheide ich vier verschiedene Aspekte von Akteurschaftszuschreibungen: Akteursbenennung sowie die Zuschreibung von Zielen, Handlungsmitteln und Motiven. Das analytische Vorgehen gestaltete sich dabei so, dass bei jedem einzelnen Beitrag ${ }^{14}$ erfasst wurde: (1) wer als verursachender und intentional handelnder Akteur bestimmt werden kann, (2) mittels welcher Handlungen er in Erscheinung tritt, (3) zu welchem konkreten Ziel bzw. Zweck der Akteur diese Handlung ausübt und (4) aufgrund welcher Motive (Interessen, Prinzipien, Werte etc.) der Akteur (laut der jeweiligen Reichsbürgergruppe) handelt. Zweierlei steht bei der Verfahrensweise im Hintergrund: Zum ersten ist es durch die ausschließliche Fokussierung auf Akteurschaftszuschreibungen möglich, verschiedene Beiträge durch ein klares Vergleichskriterium der Analyse zugänglich zu machen. Zum anderen bietet der theoretisch-deduktiv hergeleitete Fokus auf Akteurschaftszuschreibungen als Analyserahmen darüber hinaus auch genug Spielraum

\footnotetext{
13 Bezüglich der Auswahlstrategie wurde wie folgt vorgegangen: Anhand der Berichte der deutschen Verfassungsschutzbehörden aus den Jahren 2016 bis 2018 (vgl. BMI 2016, 2017, 2018) wurden alle Gruppen zusammengetragen, die den Kriterien der „Reichsideologie“ gemäß Stöss’ Definition folgen, sich gleichzeitig in ihrer Argumentation auf ganz Deutschland beziehen und einen abrufbaren Internetauftritt aufweisen. So konnte sichergestellt werden, dass relevante und überregional auftretende Gruppen Berücksichtigung finden, und zugleich dem Umstand Rechnung getragen werden, dass das Internet ein wichtiges Kommunikationsmedium der Szene darstellt (vgl. Rathje 2017a). Des Weiteren wurden in einem zweiten Schritt auch aus Qualitätszeitungen und wissenschaftlichen Publikationen weitere Gruppen erhoben, sofern dort thematisierte Gruppen den oben ausgeführten Kriterien entsprachen. Auf diese Weise konnte ein Korrektiv zu den Berichten der Verfassungsschutzbehörden gebildet werden, da auch berücksichtigt werden musste, dass behördliche Publikationspraxen sich an anderen Kriterien und Sachzwängen orientieren, als es für eine soziologische Analyse notwendig ist.

${ }^{14}$ Hierunter sind eigenständige, von einer Reichsbürgergruppe selbst verfasste und online veröffentlichte Texteinheiten zu verstehen, die einen eigenen Link aufweisen.
} 
für eine inhaltliche, induktiv-explorative Untersuchung konkreter Zuschreibungen (vgl. Kelle und Kluge 2010; Ullrich 2020) ${ }^{15}$. Auf diesem Weg konnten so insgesamt 1394 Akteurskonstruktionen als In-Vivo-Codes bestimmt werden, die weitergehend untereinander inhaltlich-vergleichend in Beziehung gesetzt werden konnten. Hierbei wurden in einem ersten Schritt Oberkategorien für inhaltlich ähnliche Akteurskonstruktionen bei jeder untersuchten Reichsbürgergruppe aus den In-Vivo-Codes entwickelt (vgl. Kelle und Kluge 2010). Diese Oberkategorien wurden im Weiteren zwischen den Gruppen verglichen, wobei durch dieses induktive Vorgehen fünf zentrale Themenfelder bestimmbar waren. Auch die inhaltliche Relevanz religiöser Semantiken konnte auf Basis dieses Typisierungsprozesses explorativ herausgearbeitet werden: Religiöse Semantiken wurden hierbei jedoch nicht als eigenständiges Themenfeld ${ }^{16}$ typologisiert. Dies liegt darin begründet, dass religiöse Akteurschaftszuschreibungen quer zu den fünf zentralen Themenkomplexen liegen und innerhalb dieser Komplexe thematisiert werden.

Eine Zusammenfassung der Typologisierung zeigt folgendes Bild: Grundsätzlich kann die Argumentation der Reichsbürger als eine naturalisierende Perspektive auf die (soziale) Welt begriffen werden, die die fünf Themenfelder einklammert. (1) Deutschland wird im Narrativ der Reichsbürger in einer naturalisierenden und ethnozentrischen Perspektive wahrgenommen, in der dem Land eine globale Sonderstellung im Kontext einer umfassenden Verschwörung zukommt. (2) Die Akteure, die hinter dieser Verschwörung vermutet werden, verweisen inhaltlich auf klassische antisemitische, antizionistische und antiamerikanische Zuschreibungen. Das Eigene, das als Teil einer natürlichen Ordnung begriffen wird, wird in Abgrenzung zu jüdischen, zionistischen und amerikanischen Akteuren, die als künstlich und bedrohlich verstanden werden, thematisiert. (3) Eine solche Gegenüberstellung findet darüber hinaus auch in rechtlicher Hinsicht statt, da den Deutschen und auf kollektiver Ebene dem Deutschen Reich quasi-naturhafte Rechte zugeschrieben werden, die wiederum äußerlich von verschwörerischen Praktiken bedroht erscheinen. (4) Dies geht damit einher, dass sich die Reichsbürger zuschreiben, eine überlegene Einsicht in die natürliche Ordnung der Welt zu besitzen, die es ihnen ermöglicht, die Lügen und manipulativen Bestrebungen der Verschwörer zu durchschauen und diese, analog einer populistischen Medien- und Elitenkritik, abzuwerten. (5) Die vorherigen Zuschreibungen führen für die Reichsbürger zu der kämpferischen Forderung, Souveränität $\mathrm{zu}$ erhalten und zu verteidigen. Dies impliziert, einen durch die verschwörerische Willkürherrschaft vermeintlich herbeigeführten Ausnahmezustand im Namen der kollektiven wie individuellen Freiheit zu beenden. Hierbei zeigt sich, dass religiöse Semantiken gerade eine legitimierende Zuschreibung der eigenen Position beinhalten, die überdies Religion oft als Teil einer ahistorisch bestimmten Identität versteht.

\footnotetext{
15 In anderer Hinsicht weisen Witzel (2000) und Ullrich (2020) in Bezug auf qualitative Interviews darauf hin, dass der Forschungsprozess als ,induktiv-deduktives Wechselverhältnis“ (Witzel 2000, S. 3) angelegt sein sollte.

16 Die Verdichtung der empirisch vorliegenden Zuschreibungen zu Themenfeldern setzte zentral an der Herausarbeitung sich über mehrere der vier Dimensionen des Akteurschaftsverständnisses erstreckenden Zuschreibungen an - hier zeigt sich, dass religiöse Semantiken verstärkt im Bereich der legitimierenden Zuschreibungen anzutreffen sind und weniger kohärente und Akteursdimensionen übergreifende Muster erkenntlich waren.
} 
Im Folgenden wird daher gezeigt, inwieweit diese naturalisierenden Zuschreibungen eine ,religiös-(über-)natürliche Dimension“ aufweisen. Um diese religiöse Dimension der reichsbürgerlichen Argumentation herauszuarbeiten, nehme ich bei der Darstellung primär auf Textstellen Bezug, die als charakteristisch für die induktiv entwickelten Themenfelder im Zusammenhang mit religiösen Semantiken gelten können.

\subsection{Zentrierung auf Deutschland}

Es ist wenig verwunderlich, dass ein wichtiger Themenkomplex für die zwölf Reichsbürgergruppen eine starke Fokussierung auf Deutschland ist. Es können hier sowohl Zuschreibungen auf Deutschland bzw. die Deutschen oder das Deutsche Reich als Form eigener Akteurschaft als auch Zuschreibungen, dass Verschwörer die derzeitige Bundesrepublik Deutschland als Instrument nutzen, als relevante Akteurschaftskonstruktionen ausgemacht werden. Hierbei erscheinen „die Deutschen“ als natürliches Kollektiv, dem das künstliche Konstrukt der BRD gegenübergestellt wird $^{17}$.

In ähnlicher Weise artikuliert sich diese „Überhöhung“ der Deutschen auch in religiösen Kategorien - so beispielsweise beim „Deutschen Kolleg“, einer sowohl reichsbürgerlichen Semantiken wie auch dem Nationalsozialismus nahestehende Gruppe: „Das Reich als Schwert Gottes, als Katechont und Wahrer der kosmischen Ordnung in Europa und auf dem Erdenrund. Von den Sternenmänteln der deutschen Kaiser bis zum gestirnten Himmel über Kants Königsberg war der deutsche Glaube immer kosmozentrisch und damit Haßobjekt aller anthropozentrischen Kulte einschließlich des Christentums“ (Deutsches Kolleg 2019a). In Übereinstimmung mit der „Europäischen Aktion“, die eine „Ostara-Feier“ als „Gegenpol zur christlich-liberal-dekadenten Spaß- und Konsumgesellschaft" (Europäische Aktion 2019a) stilisiert, wird den Deutschen sowie ihrem „Reich“ eine herausgehobene Stellung zugesprochen. Diese Stellung richtet sich ferner gegen eine mit Christentum und Moderne assoziierte Vorstellung bundesdeutscher Kultur. Das „Reich“ steht hierbei dafür ein, dass zu einer kosmischen - und in diesem Sinne ontologisch ausgezeichneten und ursprünglichen - Ordnung zurückgekehrt werden soll beziehungsweise diese gegen einen möglichen apokalyptischen Untergang zu verteidigen ist.

Der Gedanke einer religiös begründeten Rückkehr zeigt sich auch bei der „ExilRegierung Deutsches Reich“, die neben dem „Reich“ außerdem die deutsche Sprache als zentralen Bezugspunkt bestimmt: „Die Deutsche Sprache spiegelt einen wirklichen und wahrhaftigen großen Geist wider. In diesem finden wir die Strukturen der Naturverbundenheit und das tiefe Wissen um Gerechtigkeit und soziales Miteinander. Diese Sprache trägt in sich die Göttlichkeit, das Göttliche, den Ur-Gott. Keine andere Sprache der Welt als die der Deutschen hat ihre Wurzeln der Ursprache noch erhalten können“ (Exil-Regierung Deutsches Reich 2019a). Dies ist insofern

\footnotetext{
17 Hierin spiegelt sich somit auch das weiter oben thematisierte Konzept der Reichsideologie wieder: Die von verschwörerischen Akteuren gelenkte BRD verweist auf die Illegalität und Illegitimität des deutschen Staates, während sich die Identifikation der eigenen Akteurschaft mit dem Deutschen Reich auf dessen Fortexistenz bezieht (vgl. Rathje 2017a; Stöss 2010).
} 
als Rückkehr zu bestimmen, als dass die „Exil-Regierung Deutsches Reich“ einen zunehmenden „Verfall“ der deutschen Sprache, der durch verschwörerische Tätigkeiten hervorgerufen werde, aktiv zu verhindern und umzukehren versucht. Anders als in den vorherigen Stellen wird hier wiederum undifferenziert von Göttlichkeit gesprochen, sodass weniger ein konkretes begriffliches Konzept im Vordergrund steht als vielmehr eine diffuse, religiöse „Ursprünglichkeitsassoziation“ hervorgerufen wird $^{18}$.

Dass Religion eher in einem unspezifischen Sinne verwendet wird, wird auch an einer anderen Passage vom „,Deutschen Kolleg“ deutlich. Konträr zur Abwertung des Christentums im obigen Zitat bezieht sich dieses mit dem Versuch einer Analogisierung von Jesus, Adolf Hitler und Rudi Dutschke in positiver Hinsicht auf das Christentum: ,Wie bei Jesus von Nazareth erkennt bei Rudi von Luckenwalde seine Gemeinde erst geraume Zeit nach seinem Tode, daß ihr ein heilsgeschichtliches Individuum begegnet war. (...) Hitler und Dutschke waren die beiden charismatischen Führer, die das Deutsche Volk im 20. Jahrhundert hervorgebracht hatte: Hitler der Führer der großen deutschen Arbeiter- und Volksbewegung von 1933, Dutschke der Führer der kleinen deutschen Studentenbewegung von 1968. (...) Diese Wiederauferstehung Dutschkes offenbarte, daß die Vorsehung das Heil - schon zum zweiten Male im 20. Jahrhundert - auf einen Erlöser der Deutschen gelegt hatte"19 (Deutsches Kolleg 2019b). Neben einem expliziten Anschluss an christliche Metaphorik wird hier darüber hinaus deutlich, inwiefern ein christlich-messianischer Erlösungsglaube für das auf Deutschland fokussierende Narrativ der Reichsbürger nützlich gemacht wird: Ein nationaler Führer soll auf Basis der Vorsehung die Deutschen erlösen - sprich: das Motiv einer transzendenten Erlösung übersetzt sich säkularisiert in die Erlösung eines nationalen Kollektivs in der Immanenz. Wenn auch mit starker Zuspitzung auf die verschwörungssemantische Dimension, können nationale Erlösungsvorstellungen auch in anderen Teilen des Rechtsextremismus vorgefunden werden (vgl. Schuppener 2008).

\subsection{Antisemitismus}

Wie schon von Rathje (2021) herausgearbeitet, hat sich auch bei der Untersuchung von Akteurschaftszuschreibungen gezeigt, dass Antisemitismus eine wichtige Argumentationslinie der Reichsbürger darstellt. Dies äußert sich dabei so, dass ,den Juden“ eine übermächtige - potenziell weltkontrollierende - verschwörerische Handlungsmacht zugeschrieben wird. Diese drückt sich oftmals in chiffrierter Form aus, sodass an die Stelle eines direkten Bezuges auf die subjektiv projizierte Macht

\footnotetext{
18 Die thematische Fokussierung auf das Deutsche Reich sowie die Rettung der deutschen Sprache knüpfen an verbreitete Motive rechtsextremer Diskurse an, wobei die Reichsbürgergruppe gerade deren verschwörungssemantischen Gehalt betonen.

19 An dieser Stelle muss kontextualisierend darauf hingewiesen werden, dass unter anderem der frühere Anwalt der linken, außerparlamentarischen Opposition von 1968 und RAF-Terrorist Horst Mahler, der sich später dem Rechtsextremismus zuwandte und als verurteilter Holocaustleugner eine Gefängnisstrafe ableistete, Teil des „Deutschen Kollegs“ ist und an den Beiträgen dieser Gruppe mitgewirkt hat. Der positive Bezug auf Dutschke und die Studierendenbewegung ist in diesem Sinne für die Gruppe charakteristisch und lässt sich nicht anhand von Beiträgen anderer Gruppen rekonstruierten.
} 
der Juden mit dieser assoziierbare Platzhalter gesetzt werden (vgl. Salzborn 2020; Rensmann 2001; Holz 2010; Beyer und Krumpal 2010). Neben einem oftmals thematisierten Bezug zur finanziellen Macht ,der Juden“, der sich unter anderem durch die Zuschreibung von Akteurschaft auf die Rothschilds oder George Soros zeigt, werden auch religiös konnotierte Chiffren in diesem Kontext angesprochen. Mit solchen Bezügen kann ferner auch die inhaltliche Nähe zu anderen rechtsextremen Strömungen unterstrichen werden, die ähnliche Motive aufgreifen.

Erneut kann hier die Gruppe „Deutsches Kolleg“ angeführt werden, die nicht nur häufig auf antisemitische Zuschreibungen zurückgreift, sondern sich auch durch einen religiös gefärbten Antisemitismus definiert: „Zu Kants Zeiten war das absolute Böse als deuteronomistisches Völkermordgebot der mosaischen Religion, das damals sich in Frankreich im terreur austobte, bekannter als heute, weil man noch die Bibel las; heute ist das Absolute Böse in passiver Form als Holocaust-Kult im Umlauf, der seine Glaubwürdigkeit beim auserwählten Volk aus dem Wissen um die Grundform des aktiven Völkermordgebotes bezieht, das im fünften Buch Moses nur gelegentlich zum Vertreibungsgebot abgemildert ist“" (Deutsches Kolleg 2019c). Darüber hinaus heißt es an anderer Stelle: „Der Hirtenstab Abrahams und des Bischofs von Rom sind nomadisches Ursymbol und einziges, äußerst primitives Arbeitsmittel des Hirten. Dieser Stecken und Stab des Viehhirten wie des Seelenhirten ist Machtsymbol der Nomadenherrschaft (...)“(Deutsches Kolleg 2019c).

Mehrerlei ist an diesen Zuschreibungen interessant. Zum Ersten zeigt sich hier eine unmittelbare Verbindung zwischen dem christlichen Antijudaismus der Vormoderne und dem ,postchristlichen“ Antisemitismus der Moderne. „Die Juden“, umschrieben durch die Verweise auf die mosaische Religion und das Nomadentum, erscheinen als jahrhundertealtes verschwörerisches ,,absolutes Böses“ mit klarem Herrschaftsanspruch. Dieser Anspruch wird wiederum als religiös begründet begriffen - in diesem Sinne kann auch von einer antijudaistischen Anreicherung der für den modernen Antisemitismus typischen Zuschreibungen auf „,den Juden“ gesprochen werden, bei dem zwischen religiöser und säkularisierter Zuschreibung von Handlungsmacht eine gewisse Ambivalenz entsteht. Interessant ist bei dieser Zuschreibung von Handlungsmacht ferner die durch den Hirtenstab symbolisierte Verbindung von Judentum und Christentum. Bereits Sigmund Freud sah den Antisemitismus im Zusammenhang von Christentum und Judentum begründet: Dem Hass auf Jüdinnen und Juden liege eine Verschiebung zugrunde, bei der die ,schlecht getauften" Christen ihre Ablehnung auf den jüdischen Ursprung des Christentums projizieren (vgl. Freud 1999) ${ }^{20}$. Abweichend von der Verschiebungsthese findet hier eher eine direkte symbolische Identifikation von Judentum und Christentum statt, da beide als „,nomadisch“ und damit als Teil einer „,entwurzelten“ Welt gelten, sodass das antisemitische Stereotyp hier das Christentum als jüdisch und folglich als verschwörerisch begreift. Die Umschreibung durch den Begriff des „Nomadentums“ geht dementsprechend mit - auch an anderen Stellen angesprochenen - Assozia-

\footnotetext{
20 Auch Ernst Simmel thematisiert in seinem Aufsatz „Antisemitismus und Massen-Psychopathologie“ den antisemitischen Bezug der Christen auf das Judentum, indem er herausarbeitet, dass die ,den Juden“ zugeschriebenen Morde an christlichen Kindern projektiv in Verbindung mit der Beschuldigung der Ermordung Jesus stehen (vgl. Simmel 1993).
} 
tionen von Juden als „wurzellos“ und „heimatlos“ einher. Diese Assoziation steht in einem engen Zusammenhang mit dem zugeschriebenen Gegensatz zwischen einem als natürlich imaginierten - eigenen - (deutschen) Volk und dem Judentum. Während sich das erstere Kollektiv bei den Reichsbürgern durch Bezug zu Natur und Boden ,,auszeichnet“, gilt ihnen letzteres als antinatürliches Kollektiv, das das völkische Volksprinzip in Frage stellt (vgl. Holz 2010).

Zum Zweiten zeigt sich, dass nicht nur der „nomadische Herrschaftsanspruch“ religiös begründet wird. Auch das im modernen Antisemitismus oft verwendete Motiv der Umkehr von Opfer und Täter erfährt eine religiöse Fundierung, indem dem Judentum ein „Völkermordgebot“ zugeschrieben wird.

Zum Dritten ist so erkenntlich, dass die verschwörerische Akteurschaft „,der Juden“ dabei nicht nur als wirkmächtig, sondern auch als seit Jahrhunderten wirksam begriffen wird, insofern das Judentum sowohl für den ,großen Terror“ im revolutionären Frankreich als auch aktuell für einen vermeintlichen „Holocaust-Kult““ verantwortlich gemacht wird. Die schuldabwehrende Zuschreibung auf „die Juden“ wird auch hier mit religiösen Semantiken verbunden. Die Handlungen gegen nichtjüdische Menschen werden von den Reichsbürgern als Konsequenz der religiösen Schriften des Judentums begriffen. Auch in dieser Hinsicht kann so von einer Verschränkung antijüdischer mit antisemitischen Stereotypen gesprochen werden.

\subsection{Recht und Entrechtung}

Während mit dem überhöhenden Deutschlandbezug und dem Auftreten von Antisemitismus Themenfelder ausgemacht werden konnten, die auch schon an anderer Stelle zur Charakterisierung der Reichsbürgerszene herangezogen wurden (vgl. Rathje 2017a; Rathje 2021), sticht die Thematisierung von Recht und Entrechtung dadurch hervor, dass diese bisher weniger als ein verbreitetes Motiv rechtsextremer Bewegungen herausgearbeitet wurde. Dennoch zeigt sich auch hier, dass die Reichsbürgergruppen die Thematisierung von Recht in ihre naturalisierende Perspektive einbetten: Bezüglich des (deutschen) Individuums erscheint so das Menschenrecht als eine Art „außersoziale“ Tatsache jenseits aller sozialer Praxis, während analog hierzu auf Ebene des Staates das Deutsche Reich durch ein als naturhaft und ahistorisch vorgestelltes Völkerrecht legitimiert wird. Beide Formen werden wiederum als akut bedroht durch die rechtsfernen und rechtsauflösenden Handlungen der Verschwörer angesehen ${ }^{21}$. Gerade durch diese legitimierende Funktion von naturalisierten Rechten und Rechtsordnungen zeigt sich der argumentative Brückenschlag zur religiösen Dimension. So wird in einem ersten Schritt davon abgesehen, dass die Genese einer Rechtsordnung aus der Institutionalisierung sozialer Praktiken verstanden werden muss. Recht wird so als natürliches Faktum begriffen, das unabhängig von den jeweiligen Verhältnissen existieren würde. Es findet in diesem Sinne eine Hypostasierung des Rechts statt, das der Veränderung durch soziale Praxis vermeintlich entzogen ist. Bei einigen Gruppen wird Recht nun ferner in eine transzendente Sphäre verschoben. Recht ist in diesem Verständnis nicht nur einfach vorhanden,

${ }^{21}$ Für eine Kritik an naturalisierenden Rechtsvorstellungen (insbesondere des Menschenrechts), siehe: Arendt (1986) und Hunt (2018). 
sondern wird als Aspekt einer göttlichen Ordnung verstanden. Auch hier wird Recht somit losgelöst vom Sozialen und von geschichtlicher Entwicklung begriffen.

Dies zeigt sich besonders deutlich bei der Reichsbürgergruppe „Amt für Menschenrechte", die versucht, die Deutschen über vermeintlich vorenthaltene Grundrechte zu informieren. So spricht das Amt für Menschenrechte davon, dass ,humanitäre Organisationen des monotheistischen Volkes bevorrechtfähige Stiftungen im transzendenziellen Glaube (sind)“ (Amt für Menschenrechte 2019a), und sieht das deutsche Volk rechtlich im Kontext eines ,originär-bekennenden Konfessionsstaates“ (Amt für Menschenrechte 2019a). Ferner führt diese Gruppe in Bezug auf die Gründung eines eigenen „Gerichtshofes“ aus: „Absolutes Ziel des Gerichthof der Menschen ist die (...) Wahrung, Umsetzung, Förderung und (...) Schutz des Recht der Menschen nach dem Schöpferbund in Treue zum Glauben im Naturrecht (...). Der Gerichthof der Menschen sichert die Genesung des Menschen im Heiligen Auftrag der Ganzheitlichkeit, da es keine Missionierung, kein Böse und kein Gut im Recht gibt" (Amt für Menschenrechte 2019b).

An dieser Stelle ist hervorzuheben, dass hier zwar religiöse Bezüge deutlich zu erkennen sind, diese aber jenseits der Begriffsverwendung nicht unmittelbar an religiöse Konzepte anzuknüpfen scheinen. Das „Amt für Menschenrechte“ bedient sich vielmehr dieser Begriffe, ohne gleichzeitig einen konkreten Inhalt damit zu verbinden - nicht nur werden religiöse „Kunstbegriffe“ geschaffen, auch erscheint Religion im Kontext der reichsbürgerlichen Thematisierung von Recht eher als diffuser $\operatorname{Code}^{22}$ zur Legitimation der eigenen Akteurschaft und damit einhergehend der Rechtsansprüche des Volkes. Religion (wie auch die Natur) fungiert in diesem Sinne als letzte Instanz beziehungsweise als Ursprung (imaginierter) partikularer Rechte und gibt den Reichsbürgergruppen so eine Letztbegründung an die Hand, aus der sie die absolute Gültigkeit ihrer subjektiven Rechte herleiten können.

Darüber hinausgehend kann man so das religiöse Framing von Recht auch als Bridging-Prozess ${ }^{23}$ zwischen Recht, reichsbürgerlicher Verschwörungssemantik und religiöser Legitimation interpretieren. Hierbei werden zwar religiös anmutende Rechtsbegriffe aufgeworfen, sie verweisen jedoch nicht auf einen substantiellen Inhalt. Dies trifft ferner nicht nur in Bezug auf dezidiert religiöse Konzepte zu die Reichsbürgergruppen entwickeln an dieser Stelle auch kein eigenes Verständnis, sodass religiöse Rechtslegitimationen größtenteils „leere“ Ausdrücke bleiben.

\subsection{Wahrheit und Manipulation}

Eine solche Form der legitimierenden Zuschreibung, die sich eher einer unbestimmten religiösen Konnotation in der Argumentation bedient, lässt sich auch für Argumentationsmuster herausarbeiten, die das Verhältnis von Wahrheit und Manipulation

\footnotetext{
22 Siehe hierzu auch das Konzept des ,kulturellen Codes“ von Volkov (2000). Auch bei diesem steht weniger der unmittelbare Inhalt als vielmehr deren identitäre Implikationen - bzw. in den Begrifflichkeiten dieses Artikels: die Implikationen für die eigene Akteurschaft - im Vordergrund.

23 Hierunter ist ,the linkage of two or more ideologically congruent but structurally unconnected frames regarding a particular issue or problem“ (Snow et al. 1986, S. 467) zu verstehen. Dabei gilt ferner, dass ein einzelner „Frame“ mit Goffman als „schemata of interpretation“ (Goffman 1974, S. 21) anzusehen ist, wodurch Situationsdeutungen und Handlungskonsequenzen maßgeblich beeinflusst werden.
} 
in den Vordergrund stellen. Grundlegend zeigt sich bei diesem Themenkomplex, dass die Reichsbürgergruppen „,den“ Medien vorwerfen, Teil der gegen Deutschland gerichteten Verschwörung zu sein, indem sie im Sinne und als Teil „der Eliten“ die Bevölkerung bewusst manipulieren, umerziehen und systematisch belügen würden (vgl. Krüger und Seiffert-Brockmann 2018). Dem gegenüber schreiben die Reichsbürgergruppen sich selbst die Kenntnis einer überlegenen Form allgemeingültiger Wahrheit zu, die sie in aufklärerischer Absicht an das getäuschte Volk vermitteln wollen - ein Gegensatz, der allgemein auf die argumentative Nähe zwischen verschwörungssemantischen und populistischen Narrativen verweist ${ }^{24}$.

Auch hier zeigt sich die Thematisierung religiöser Semantiken darin, dass deren Gebrauch die eigene Akteurschaft legitimiert: So sind die Reichsbürgergruppen nicht nur ,göttlich berufen“, das heilige Deutschland zu verteidigen, sondern sie sehen sich zudem in der Position, trotz der Wirkmächtigkeit der verschwörerischen Manipulationen die Lügen der Verschwörer zu erkennen. Derart beanspruchen sie implizit einen vermeintlich erkenntnistheoretisch überlegenen Standpunkt: „Wie wir bereits in Erfahrung gebracht haben, gehört es zum bestimmenden Wesenskern einer jeden Ideologie, dass diese sich nicht an den heiligen Gesetzmässigen des natürlichen Schöpfungsvorgangs ausrichtet, sondern die Welt und ihre Völker vielmehr als Spielball von ausbeuterischen Geld- und Verwertungsinteressen betrachtet" (Europäische Aktion 2019b). Es wird deutlich, dass weniger auf das konkrete Verständnis der „natürlichen Schöpfung“ eingegangen wird, als dass vielmehr versucht wird, den eigenen Standpunkt assoziativ in die Nähe der Schöpfung zu rücken. Dies geschieht hier erneut gemäß der Dichotomie zwischen eigener und verschwörerischer Akteurschaft, wobei letzterer zugeschrieben wird, durch Ideologie gegen die Schöpfung zu handeln. In den Worten der Reichsbürgergruppe Staatenlos.info: „Man muss kein gläubiger Mensch sein, um zu erkennen, dass die so genannten ,Eliten“ schöpfungsfeindliche Individuen sind, die zwar in Sonntagsreden so tun, als seien sie Lichtgestalten, anhand ihrer Handlungen aber genau als das Gegenteil dessen zu erkennen sind" (Staatenlos.info 2019a).

Interessant ist hier ferner, dass der Rekurs auf „die Schöpfung“ ein zentrales Bindeglied zwischen religiösen und naturalisierenden Interpretationen der sozialen Welt darstellt. Sie kann je nach Interpretation sowohl als biologisierende Kategorie als auch als direkte Anknüpfung an christliche Motive gelesen werden. Eine solche changierende Vagheit der Bezugnahme ist kennzeichnend für den reichbürgerlichen Religionsbezug: Religiöse Terminologie wird in die reichsbürgerlichen Zuschreibungen eingebettet, wobei sie eher als Marker einer (identitären) Position - beziehungsweise als (zu den Verschwörern sich) abgrenzende Markierung im Sozialen -, denn als ausgefülltes Konzept verstanden werden muss.

\footnotetext{
24 Ähnlich wie Verschwörungssemantiken zeichnen sich auch populistische Narrative durch ein antagonistisches Weltbild aus: nicht unmittelbar zwischen eigener Akteurschaft und Verschwörern - auch wenn hier durchaus ebenfalls Verknüpfungen bestehen -, sondern zwischen einerseits den - als oftmals korrupt dargestellten - Eliten und „der“ politischen Klasse sowie andererseits dem „guten“ Volk, für das der Populismus sich als Stimme bestimmt. Eliten und Verschwörer teilen in gewisser Hinsicht oftmals gemeinsame Charakteristika, indem sie gegen die natürlichen Interessen handeln, sich selbst nur bereichern oder eine illegitime Herrschaft festigen wollen würden (vgl. Mudde 2004 und Canovan 2004).
} 
In diesem Sinne geht es weniger um Inhalte als um eine Konstruktion von kollektiver Eigenakteurschaft in Abgrenzung zu den Verschwörern. Deutlich wird dies im Weiteren auch dadurch, dass beispielsweise „Staatenlos.info“ an anderer Stelle ihren Anspruch auf Wahrheit auch in Abgrenzung zu ,den“ Weltreligionen formuliert: „Alle internationalen Ideologien- als auch diverse große, missionarische Weltreligionen kommen aus demselben Geist bzw. haben heute dieselben finanziellen Quellen! Die geistigen Theorien werden für das Machinteresse des intern. Bankenkartells und dessen Kapitäns benutzt. Beweis dazu ist die finanzielle Steuerung über das internationale Bankenkartell“" (Staatenlos.info 2019b). Nicht nur knüpfen sie hier an antisemitisch konnotierte Zuschreibungen bezüglich der Macht der Banken an; auch zeigt sich, dass Religionen, obwohl aus vorherigem Zitat eine legitimierende Funktion hervorgeht, gleichzeitig auch als Instrument vermeintlicher Verschwörer gelten können. Sofern Religion bei den Reichsbürgergruppen als ein Mittel verschwörerischer Akteure angesehen wird, wird ihr so auch eine manipulative Verwendung unterstellt.

Diese Widersprüchlichkeit kann einerseits in der Struktur von Verschwörungssemantiken begründet liegen, da sie nicht als in sich logisches und stringentes Ganzes aufgefasst werden sollten: Verschwörungssemantiken können in Bezug auf ihre unmittelbare Argumentation vielmehr Widersprüche aushalten und sie bisweilen sogar für sich nutzbar machen (vgl. auch Douglas et al. 2016; Wood et al. 2012). Gleichzeitig bietet sich andererseits auch eine weitere Lesart an - nämlich, dass hier ein Anhaltspunkt dafür vorliegen kann, dass die generelle Ambivalenz zwischen bestimmten „religiösen Traditionslinien“ innerhalb des Rechtsextremismus an dieser Stelle zu Gunsten einer natürlichkeits-, ursprungs- und schöpfungsverbundenen Position in Abgrenzung zu Religion als Institution aufgelöst wird.

\subsection{Freiheitskampf und Versklavung}

Das letzte Themenfeld fokussiert die Selbststilisierung der Reichsbürger zu Freiheitskämpfern gegen die Verschwörung, die die Deutschen und/oder die Menschheit versklaven würde. Die Argumentation der Reichsbürger weist hierbei (implizit) große Überschneidungen mit Überlegungen des nationalsozialistischen Rechtstheoretikers Carl Schmitt ${ }^{25}$ auf. Im Sinne Schmitts (1922) nehmen die Reichsbürger einen andauernden Ausnahmezustand wahr, der durch die souveräne Willkürherrschaft der Verschwörer begründet ist. Dagegen wiederum bringen die Reichsbürger eine eigene Souveränität in Stellung: Im Gegensatz zu der derzeitigen Souveränität der Verschwörer erscheint das eigene souveräne Handeln hierbei jedoch als natürlich legitimiert, während verschwörerische Formen der Souveränität als uneigentlichkünstliche Formen abgewertet werden.

Die Rolle religiöser Semantiken in diesem Kontext kann am anschaulichsten anhand einer Textpassage der „Europäische Aktion“ aufgezeigt werden. In dieser

\footnotetext{
25 Die Anknüpfbarkeit der reichsbürgerlichen Argumentation ist hierbei keineswegs als zufällig zu betrachten. Vielmehr kann argumentiert werden, dass die Figur des Ausnahmezustands und die Bestimmung des Politischen anhand der Freund-Feind-Unterscheidung bei Schmitt (1922) den späteren Argumentationen der Reichsbürger in wichtigen Aspekten vorgreift.
} 
Passage wird nicht nur das Thema des Freiheitskampfes angesprochen, sondern es wird auch deutlich, wie eng die fünf zentralen Zuschreibungsthemen einerseits und die Thematisierung von Religion andererseits miteinander verwoben sind und ineinander übergehen: „Unter dem Deckmantel von Nächstenliebe und Barmherzigkeit sollen die ethnisch-kulturelle Vielfalt vernichtet und die (Gojim-)Völker in einem multirassischen Schmelztiegel aufgelöst werden (...). Um unseren Fortbestand als Deutsche wie auch als Europäer zu sichern, müssen wir uns nicht nur physisch zur Wehr setzen, sondern auch geistig-kulturell wieder aufrichten (...). Gerade die Völker sind höchste Träger dieser göttlichen Ordnung. Ihr Dasein ist Ausdruck einer naturgesetzlich-evolutionär bedingten Menschwerdung. (...) Die aktuelle Rassen- und Kulturvermischung versündigt sich an den Naturgesetzen der göttlichen Schöpfung“ (Europäische Aktion 2019c).

Hier zeigt sich, dass sich die Überhöhung des Deutschen und das Postulat einer (wahren) göttlichen Ordnung mit antisemitischen und rassistischen Zuschreibungen im Kontext eines religiös gerahmten Freiheitskampfes argumentativ verbinden. Es ist deutlich ersichtlich, wie eng naturalisierende und religiöse Zuschreibungen miteinander verwoben werden können. Sie stellen gewissermaßen die naturalisierend-religiöse Legitimation dafür dar, sich (gewaltsam) gegen die Verschwörung zu wehren, und können so auch als eine Handlungen anleitende und zu Handlungen aufrufende Form der Legitimation gedeutet werden.

Auf anderer Ebene verbindet sich der Handlungsaufruf zur Befreiung auch mit der in rechtsextremen Bewegungen populären Verschwörungssemantik eines „Bevölkerungsaustausches“: So wird im Narrativ von Staatenlos.info der BRD als Akteur zugeschrieben, einen islamischen Staat in Deutschland aufzubauen, wobei die „einheimische deutsche Bevölkerung (...) offensichtlich zielgerichtet ausgeplündert, enteignet und existenziell vernichtet (werde)“ (Staatenlos.info 2019c). Typisch für das gesamte Material ist hierbei, dass Muslime zwar rassistisch markiert werden, jedoch selbst nicht als handelnde Akteure auftreten. Vielmehr findet eine „doppelte“ Abwertung in dem Sinne statt, als dass Muslime zwar als zu bekämpfende Bedrohung begriffen werden, selbst jedoch nur Mittel eines (hintergründig agierenden) verschwörerischen Akteurs sind, der „,den Islam“ lediglich benutzt, um die Deutschen zu versklaven und zu vernichten. In einer solchen Konstruktion verbinden sich antisemitische Akteurschaftszuschreibungen mit rassistischen Abwertungen und einem Handlungsaufruf an die Deutschen, sich sowohl gegen ,Islamisierung“ als Symptom der Verschwörung als auch gegen die eigentlich souveränen Verschwörer zu wehren.

\section{Fazit und Diskussion}

Wie dargestellt, kann Religion in zweierlei Hinsicht in Bezug auf reichsbürgerliche Verschwörungssemantiken thematisiert werden. Hieraus folgt, dass die Untersuchung von Religion in der Argumentation von Reichsbürgern einen wichtigen Zugang zum Verständnis reichsbürgerlicher Verschwörungssemantiken bereitstellt. Zum einen ist ersichtlich, dass beide als „funktionale Äquivalente“ angesehen werden können, die in Reaktion auf moderne Formen der Vergesellschaftung Komplexi- 
tät reduzieren und Sinn stiften. Zum anderen konnte ich anhand einer Akteurschaftszuschreibungen untersuchenden Inhaltsanalyse aufzeigen, dass nicht nur funktionale Gemeinsamkeiten existieren, sondern dass religiöse Semantiken auch auf inhaltlichsubstantieller Ebene Eingang in die Argumentation relevanter Reichsbürgergruppen finden. Dies kann vor allem in der Hinsicht herausgearbeitet werden, dass religiöse Semantiken in verschiedenen Themenfeldern anzutreffen sind und hierbei als legitimierende Zuschreibung ${ }^{26}$ der eigenen Akteurschaft auftreten. In diesem Sinne stellt Religion für die reichsbürgerliche Argumentation kein eigenständiges Kernthema dar, sondern kommt jeweils mit diesen Themen verwoben zur Geltung. Relevant ist hierbei, dass durch die Situierung innerhalb der Argumentationsmuster religiösen Semantiken eine Kernthemen übergreifende und verbindende Rolle zukommt. Diese Rolle von religiösen Semantiken ist hierbei nicht nur in Bezug auf Reichbürger, sondern auch weitergefasst in Bezug auf rechtsextreme Bewegungen bisher wenig beachtet worden.

Ein weiterer Zusammenhang ist darüber hinaus zwischen der allgemeinen Tendenz, Akteurschaft in der sozialen Welt in naturalisierender Weise zu deuten, und dem Aufgreifen religiöser Semantiken ersichtlich: Indem Akteurschaft durch den Rekurs auf Religion begründet wird, erscheinen transzendente Ordnungsvorstellungen als legitimierende Basis der eigenen Akteurschaft. Akteure und deren Handlungen erscheinen so weniger als historisch gewachsen und durch soziale Praxis bestimmt denn als ontologisch feststehend und ewig gegeben. Zugleich changiert eine solche Enthistorisierung von Akteurschaft in vielen Fällen zwischen naturalisierenden und „transzendentisierenden“Formen, wobei nicht selten - wenn etwa von den ,Naturgesetzen der göttlichen Schöpfung“ gesprochen wird - keine trennscharfe Abgrenzung zwischen beiden Formen vorliegt ${ }^{27}$.

In diesem Sinne stehen wenig spezifizierte religiöse Konzepte und Glaubenspraktiken im Vordergrund der Argumentation, wie die Verwendung religiöser Begriffe als (kulturelle) Marker und Symbolisierung der eigenen, ewig gegebenen Akteurschaft zeigt: Deutschland, die Deutschen und das Deutsche Reich erscheinen so als integraler Teil einer göttlichen Ordnung, die im manichäischen Weltbild der Reichsbürger durch Verschwörer bedroht ist. Im Bild der Reichsbürger über diese Verschwörer werden religiöse Semantiken lediglich im Zusammenhang mit deren unterdrückerischen Zielen relevant, wobei derartige Zuschreibungen an vielen Stellen antisemitisch konnotiert sind. Legitimiert durch diese Bedrohungslage sehen sich die Reichsbürgergruppen berufen, ihre aus der göttlichen Ordnung abgeleiteten - und so als außersozial und als transzendent erscheinenden - Rechte zu verteidigen. Darüber hinaus stilisieren sie ihre eigenen Handlungen zu einem Freiheitskampf, der aus einer quasi göttlichen Berufung heraus erfolgt und zudem religiöse Praktiken als Teil einer eigenen Identität sowie als abgrenzende Bestimmung aufgreift. Hierbei

\footnotetext{
${ }^{26}$ Hinzu tritt, dass parallel die verschwörerische Akteurschaft in religiöser und antisemitischer Hinsicht als ,jüdisch“ delegitimiert wird.

27 Eine weiterführende Analyse müsste ferner zeigen, inwieweit gleichzeitig Brüche im Verhältnis von Naturalisierung und religiösen Entgesellschaftlichungen auftreten. Dies könnte gerade vor dem Hintergrund einer ideengeschichtlichen Perspektive neuzeitlicher und moderner Natur- und Religionsverständnisse einen weiteren Analysefokus etablieren.
} 
werden sowohl christliche, nordisch-mythologisch imaginierte als auch eher unbestimmt und religiös vage bleibende Begriffe verwendet.

Im Kontext der zusammenfassenden Systematisierung der Verknüpfung von religiösen Semantiken und Verschwörungssemantiken sei weitergehend darauf verwiesen, dass sich ein solcher Zusammenhang insbesondere bei Reichsbürgergruppen gezeigt hat, die stärker als andere einem völkisch-antisemitischen Weltbild anhängen zu nennen sind hier gerade das „Deutsche Kolleg“ und die „Europäische Aktion“. Eine mögliche Erklärung könnte darin bestehen, dass solche Gruppierungen deutlicher und direkter ein naturhaft-ontologisch feststehendes - eben völkisches - Bild der eigenen Akteurschaft zeichnen und zugleich einen antisemitisch-manichäischen (endzeitlichen) Kampf betonen.

Bezieht man in einem weiteren Schritt die legitimierende Rolle religiöser Semantiken wieder zurück auf den eingangs thematisierten Befund, dass derartige Semantiken trotz Relevanz bisher eher unterbelichtet geblieben sind, unterstützt die hier durchgeführte Analyse diese Relevanzbetonung: Obwohl religiöse Zuschreibungen in verschiedener Hinsicht in Argumentationen der Reichsbürgergruppen auftauchen, wurde gerade deren legitimierende Funktion bisher kaum systematisch untersucht. Religiöse Zuschreibungen können in diesem Sinne als eine wichtige Ausprägung der generellen Tendenz, Akteurschaft in enthistorisierter Form aufzufassen, verstanden werden - es findet eine Supernaturalisierung des Sozialen statt.

Die empirische Relevanzbestimmung ist hier ferner natürlich im Kontext des inhaltsanalytischen Vorgehens zu sehen. Indem ich reichsbürgerliche Verschwörungssemantiken operational als Akteurschaftszuschreibungen aufgefasst und hieraus folgend legitimierende Zuschreibungen als eine zentrale Bestimmung von Akteurschaft angesehen habe, konnten religiöse Semantiken dementsprechend auch nur im Zusammenhang mit der Untersuchung von Akteurschaftszuschreibungen erfasst werden. Andere Formen der Thematisierung von Religion, auch jenseits einer Fokussierung auf Onlinebeiträge, gingen so nicht in die Analyse ein und sind weiterhin zu analysieren ${ }^{28}$. Eine weitergehende Analyse kann jedoch durch die hier ausgearbeiteten Bestimmungen wichtige Impulse erhalten, zumal die vollständige Erhebung aller Onlinebeträge von zwölf zentralen Reichsbürgergruppen als belastbarer Ausgangspunkt angesehen werden kann.

Zugleich verweist die Analyse im Kontext einer umfassenderen Perspektive auf den Zusammenhang zwischen Religion und Rechtsextremismus. Zwar kann die Reichsbürgerszene nicht unumwunden als ,,repräsentativ“ für rechtsextreme Bewegungen gelten, da sie sich durch eine eigene Spezifik auszeichnet. Es liegt dennoch der Schluss nahe, dass aufgrund vielfältiger argumentativer Überschneidungen die hier dargestellten Einsichten weiterführend auch in Hinsicht auf deren Rolle bei anderen Strömungen des Rechtsextremismus mit einbezogen werden können. Dies erscheint nicht zuletzt dahingehend relevant, als dass in der Inhaltsanalyse erneut beobachtet werden konnte, dass religiöse Vorstellungen seit Längerem rechtsextreme

\footnotetext{
28 Als Anknüpfungspunkt für eine weiterführende Untersuchung des Stellenwerts von Religion ist zentral auch nach dem Zusammenhang zwischen religiösen Semantiken in der Reichsbürgerszene und deren sektenartigen Organisationsformen zu fragen - ein Aspekt, der in diesem Beitrag zugunsten der Fokussierung auf die inhaltlich-organisatorische Ebene analytisch zurückgestellt wurde.
} 
Weltbilder auf verschiedene Weisen prägen: Gerade die bisherigen Befunde dieses Zusammenhangs, hier dargestellt in Kap. 3, konnten so auch durch die Analyse empirisch weitergehend unterfüttert werden.

Abschließend ist zu konstatieren, dass reichsbürgerliche Verschwörungssemantiken (und damit verbunden ihre religiösen Deutungen) zunehmend auch abseits rechtsextremer Foren, Blogs und Websites anzutreffen sind und an unterschiedlichen Diskursen im gesellschaftlichen Mainstream anknüpfen. Gerade die enge Verwobenheit zwischen Religion als funktionalem Äquivalent und den Verknüpfungen auf inhaltlicher Ebene lassen befürchten, dass die derzeit beobachtbare Dynamik zwischen Verschwörungssemantiken, Religion und Rechtsextremismus sich in Zukunft noch weiter verstärken wird und dementsprechend vermehrter Aufmerksamkeit bedarf.

Funding Open Access funding enabled and organized by Projekt DEAL.

Open Access Dieser Artikel wird unter der Creative Commons Namensnennung 4.0 International Lizenz veröffentlicht, welche die Nutzung, Vervielfältigung, Bearbeitung, Verbreitung und Wiedergabe in jeglichem Medium und Format erlaubt, sofern Sie den/die ursprünglichen Autor(en) und die Quelle ordnungsgemäß nennen, einen Link zur Creative Commons Lizenz beifügen und angeben, ob Änderungen vorgenommen wurden.

Die in diesem Artikel enthaltenen Bilder und sonstiges Drittmaterial unterliegen ebenfalls der genannten Creative Commons Lizenz, sofern sich aus der Abbildungslegende nichts anderes ergibt. Sofern das betreffende Material nicht unter der genannten Creative Commons Lizenz steht und die betreffende Handlung nicht nach gesetzlichen Vorschriften erlaubt ist, ist für die oben aufgeführten Weiterverwendungen des Materials die Einwilligung des jeweiligen Rechteinhabers einzuholen.

Weitere Details zur Lizenz entnehmen Sie bitte der Lizenzinformation auf http://creativecommons.org/ licenses/by/4.0/deed.de.

\section{Literatur}

\section{Verwendete Literatur}

Adensamer, Angelika. 2017. Überschießende Kriminalisierung als Gefahr für die Demokratie: Kritik an § 247a StGB ,Staatsfeindliche Bewegung“. Juridikum 2:149-151.

Anton, Andreas. 2011. Unwirkliche Wirklichkeit. Zur Wissenssoziologie von Verschwörungstheorien. Berlin: Logos.

Anton, Andreas, Michael Schetsche, und Michael K. Walter. 2014. Einleitung: Wirklichkeitskonstruktion zwischen Orthodoxie und Heterodoxie - zur Wissenssoziologie von Verschwörungstheorien. In Konspiration. Soziologie des Verschwörungsdenken, Hrsg. Andreas Anton, Michael Schetsche, und Michael K. Walter, 9-26. Wiesbaden: VS.

Arendt, Hannah. 1986. Elemente und Ursprünge von totaler Herrschaft. München: Piper.

Baeck, Jean-Philipp. 2017. Wenn er König von Deutschland wär': Peter Fitzek und sein Imperium in Wittenberg. In Reichsbürger. Die unterschätzte Gefahr, Hrsg. Andreas Speit, 62-78. Berlin: Ch. Links.

Barkun, Michael. 2003. A Culture of Conspiracy. Apocalyptic Visions in Contemporary America. Berkeley, Los Angeles \& London: University of California Press.

Begrich, David, und Andreas Speit. 2017. „Heiliges Deutsches Reich“: Reichsidee und Reichsideologie der extremen Rechten. In Reichsbürger. Die unterschätzte Gefahr, Hrsg. Andreas Speit, 62-78. Berlin: Ch. Links.

Beyer, Heiko, und Ivar Krumpal. 2010. „Aber es gibt keinen Antisemitismus mehr“: Eine experimentelle Studie zur Kommunikationslatenz antisemitischer Einstellungen. Kölner Zeitschrift für Soziologie und Sozialpsychologie 62:681-705. 
Bischof, Susann. 2017. Männersache? Gender, Reichsbürgerinnen und Reichsbürger. In Reichsbürger. Die unterschätzte Gefahr, Hrsg. Andreas Speit, 144-158. Berlin: Ch. Links.

Bischoff, Sebastian, und Sina Arnold. 2017. Ein Wir geht immer. Nationale Ursprungsmythen im Rechtspopulismus und in der Neuen Rechten. http://beratungsnetzwerk-sachsen-anhalt.de/images/docs/ Publikationen/2017_IDA_NationalismusKritik.pdf\#page=81. Zugegriffen: 20. Aug. 2020.

Bundesministerium des Innern, für Bau und Heimat. 2016. Verfassungsschutzbericht 2016. https://www. verfassungsschutz.de/de/oeffentlichkeitsarbeit/publikationen/verfassungsschutzberichte/vsbericht2016. Zugegriffen: 9. Juli 2019.

Bundesministerium des Innern, für Bau und Heimat. 2017. Verfassungsschutzbericht 2017. https://www. verfassungsschutz.de/de/oeffentlichkeitsarbeit/publikationen/verfassungsschutzberichte/vsbericht2017. Zugegriffen: 9. Juli 2019.

Bundesministerium des Innern, für Bau und Heimat. 2018. Verfassungsschutzbericht 2018. https://www. verfassungsschutz.de/de/oeffentlichkeitsarbeit/publikationen/verfassungsschutzberichte/vsbericht2018. Zugegriffen: 9. Juli 2019.

Bundesministerium des Innern, für Bau und Heimat. 2019. „Reichsbürger“ und „Selbstverwalter“ - eine zunehmende Gefahr? https://www.bmi.bund.de/SharedDocs/topthemen/DE/topthema-reichsbuerger/ topthema-reichsbuerger.html;jsessionid=B032ECE7876C825D704106ED0B510180.1_cid287. Zugegriffen: 14. Dez. 2019.

Buschbom, Jan. 2017. Denk- und Kommunikationsstrukturen in ideologisierten Milieus: Rechtsextremismus und Islamismus. In Politischer Extremismus im Vergleich. Beiträge zur politischen Bildung, Hrsg. Ralf Altendorf, Sarah Bunk, und Melanie Piepenschneider, 211-236. Münster: LIT.

Butter, Michael. 2014. Konspirationistisches Denken in den USA. In Konspiration. Soziologie des Verschwörungsdenkens, Hrsg. Andreas Anton, Michael Schetsche und Michael K. Walter, 259-276. Wiesbaden: Springer VS.

Butter, Michael. 2018. „Nichts ist wie es scheint“. Über Verschwörungstheorien. Berlin: Suhrkamp.

Canovan, Margaret. 2004. Populism for political theorists? Journal of Political Ideologies 9:241-252.

Cantzen, Rolf. 2015. German, Götter und Gelehrte. Zu völkischen Denkmustern und Deutungsschemata vom Deutschen Kaiserreich bis heute. In Dominanzkultur reloaded. Neue Texte zu gesellschaftlichen Machtverhältnissen und ihren Wechselwirkungen, Hrsg. Iman Attia, Swantje Köbsell, und Nivedita Prasad, 59-74. Bielefeld: transcript.

Caspar, Christa, und Reinhard Neubauer. 2015. Durchs wilde Absurdistan: Was zu tun ist, wenn „Reichsbürger“ und öffentliche Verwaltung aufeinandertreffen. In „Reichsbürger“. Ein Handbuch, Hrsg. Dirk Wikling, 93-174. Potsdam: Brandenburgische Universitätsdruckerei.

Cubitt, Geoffrey. 1989. Conspiracy myths and conspiracy theories. Journal of Anthropological Society of Oxford 20:12-26.

Douglas, Karen M., Robbie M. Sutton, Mitchell J. Callan, Rael J. Dawtry, und Annelie J. Harvey. 2016. Someone is pulling the strings: hypersensitive agency detection and belief in conspiracy theories. Thinking \& Reasoning 22:57-77.

Douglas, Karren M., Joseph E. Uscinski, Robbie M. Sutton, Aleksandra Cichocka, Turkay Nefes, Chee Siang Ang, und Farzin Deravi. 2019. Understanding conspiracy theories. Political Psychology 40:3-35.

Durkheim, Émile. 1999. Über soziale Arbeitsteilung. Studie über die Organisation höherer Gesellschaften. Frankfurt am Main: Suhrkamp.

Freud, Sigmund. 1999. Der Mann Moses und die monotheistische Religion. In Schriften über die Religion Gesammelte Werke, Bd. XVI, 101-246. Frankfurt am Main: S. Fischer.

Goffman, Erving. 1974. Frame analysis. Cambridge: University Press.

Greve, Jens. 2012. Praxis - Zuschreibung - Objektivität: Argumente gegen einen reduktionistischen Individualismus und ihre Kritik. Kölner Zeitschrift für Soziologie und Sozialpsychologie 64:431-454.

Haase, Anna-Maria. 2018. „Reichsbürger und Selbstverwalter“ im Kontext politisch motivierter Gewalt in Sachsen. In Totalitarismus und Demokratie, Bd. 15, 47-71. Dresden: Hannah-Arendt-Institut für Totalitarismusforschung.

Harder, Bernd. 2018. Verschwörungstheorien. Ursachen - Gefahren - Strategien. Aschaffenburg: Alibri. Hardinghaus, Winfried. 2016. Reichsbürger und Germanische Medizin. Der Klinikarzt 45:579-626.

Helfferich, Cornelia. 2012. Einleitung: Von roten Heringen, Gräben und Brücken. Versuche einer Kartierung von Agency-Konzepten. In Agency: Die Analyse von Handlungsfähigkeit und Handlungsmacht in qualitativer Sozialforschung und Gesellschaftstheorie, Hrsg. Stephanie Bethmann, Cornelia Helffrich, Heiko Hoffmann, und Debora Niermann, 9-39. Weinheim \& Basel: Beltz Juventa.

Heller, Friedrich Paul. 2002. Mythologie und Okkultismus bei deutschen Rechtsextremen. In Handbuch Rechtsradikalismus, Hrsg. Thomas Grumke, Bernd Wagner, 203-212. Opladen: Leske und Burdrich. 
Holz, Klaus. 2010. Nationaler Antisemitismus. Wissenssoziologie einer Weltanschauung. Hamburg: Hamburger Edition.

Hoyningen-Huene, Stefan. 2003. Religiosität bei rechtsextrem orientierten Jugendlichen. Münster: LIT.

Hunt, Alastair. 2018. Wessen Recht? In Vom Recht, Rechte zu haben, Hrsg. Stephanie DeGooyer, Alastair Hunt, Lida Maxwell, und Samuel Moyn, 101-143. Hamburg: HIS.

Jansen, Frank, und Wiebke Fröhlich. 2016. „Reichsbürger“ schließt Polizisten nieder. https://www. tagesspiegel.de/politik/georgensgmuend-reichsbuerger-schiesst-polizisten-nieder/14708384.html. Zugegriffen: 15. Dez. 2019.

Keil, Jan-Gerrit. 2018. „Reichsbürger“ und Selbstverwalter - (k)ein Problem der Jugend? Zeitschrift für Jugendkriminalrecht und Jugendhilfe 2:126-131.

Kelle, Udo, und Susann Kluge. 2010. Vom Einzelfall zum Typus Fallvergleich und Fallkontrastierung in der qualitativen Sozialforschung. Wiesbaden: VS.

Kellershohn, Helmut. 1998. Völkischer Nationalismus und seine Kernideologeme. Ein knappe Begriffsklärung. http://diss-duisburg.de/Internetbibliothek/Buecher/Der\%20Spuk\%20ist\%20nicht\%20vorbei. pdf. Zugegriffen: 20. Aug. 2020.

Kron, Thomas, und Lars Winter. 2009. Aktuelle soziologische Akteurtheorien. In Handbuch Soziologische Theorien, Hrsg. Georg Kneer, Markus Schroer, 41-66. Wiesbaden: Springer VS.

Krüger, Uwe, und Jens Seiffert-Brockmann. 2018. „Lügenpresse“ - Eine Verschwörungstheorie? Hintergründe, Ursachen, Auswege. In Nachrichten und Aufklärung: Medien- und Journalismuskritik heute: 20 Jahre Initiative Nachrichtenaufklärung, Hrsg. Hektor Haarkötter, Jörg Uwe Nieland, 67-88. Wiesbaden: Springer VS.

Luhmann, Niklas. 2005. Soziologische Aufklärung 2: Aufsätze zur Theorie der Gesellschaft. Wiesbaden: Springer VS.

Marx, Karl. 2009. Das Kapital: Kritik der politischen Ökonomie. Köln: Anaconda.

Mudde, Cas. 2004. The populist zeitgeist. Government and Opposition 39:542-563.

Müller, Dieter, und Adolf Rebler. 2019. Die fahrerlaubnisrechtliche Behandlung der „Reichsbürger“. Neue Zeitschrift für Verkehrsrecht 3:119-125.

Pilz, Michael. 2014. Xavier Naidoo, der Prophet des rechten Glaubens. https://www.welt.de/kultur/pop/ article133167852/Xavier-Naidoo-der-Prophet-des-rechten-Glaubens.html. Zugegriffen: 17. Apr. 2021.

Quent, Matthias. 2015. Einleitung. In Rechtsextremismus in lokalen Kontexten. Vier vergleichende Fallstudien, Hrsg. Matthias Quent und Peter Schulz, 9-12. Wiesbaden: Springer VS.

Rathje, Jan. 2017a. „Reichsbürger“; Verschwörungsideologie mit deutscher Spezifik. http://www.sekten watch.de/drupal/sites/default/files/files/reichsbuerger_verschwoerungstheorien16.pdf. Zugegriffen: 17. Dez. 2019.

Rathje, Jan. 2017b. Die vermeintlichen „Mächte im Hintergrund“. Antisemitismus im Milieu von Reichsbürgern, Selbstverwaltern und Souveränisten. In Reichsbürger. Die unterschätzte Gefahr, Hrsg. Andreas Speit, 133-143. Berlin: Ch. Links.

Rathje, Jan. 2021. For reich and Volksgemeinschaft - against the world conspiracy: antisemitism and sovereignism in the Federal Republic of Germany since 1945. Antisemitism Studies 5:100-139.

Rensmann, Lars. 2001. Kritische Theorie über den Antisemitismus. Studien zu Struktur, Erklärungspotential und Aktualität. Berlin: Argument.

Salzborn, Samuel. 2015. Religionsverständnisse im Rechtsextremismus. Eine Analyse am Beispiel des neurechten Theorieorgans Sezession. In Jahrbuch Öffentliche Sicherheit 2014/2015, Hrsg. Martin H.W. Möllers, Robert Chr van Ooyen. Frankfurt: Verlag für Polizeiwissenschaft.

Salzborn, Samuel. 2020. Globaler Antisemitismus. Eine Spurensuche in den Abgründen der Moderne. Weinheim: Beltz.

Schmitt, Carl. 1922. Politische Theologie. Vier Kapitel zur Lehre von der Souveränität. Berlin: Duncker \& Humblot.

Schuppener, Georg. 2008. Sprache des Rechtsextremismus. Spezifika der Sprache rechtsextremistischer Publikationen und rechter Musik. Leipzig: Edition Hamouda.

Schwarzer, Matthias. 2020. Die Karriere von Xavier Naidoo ist am Ende - aber warum erst jetzt? https:/www.rnd.de/promis/die-karriere-von-xavier-naidoo-ist-am-ende-aber-warum-erst-jetztCYPZX7APSVDRBF2JMTMINE2IYQ.html. Zugegriffen: 19. Apr. 2021.

Simmel, Ernst. 1993. Antisemitismus und Massen-Psychopathie. In Antisemitismus, Hrsg. Ernst Simmel, 58-100. Frankfurt am Main: Fischer.

Snow, David A., E. Burke Rochford Jr., Steven K. Worden und Robert Bendford. 1986. Frame Alignment Processes, Micromobilization and Movement Participation. American Sociological Review 51:464-481. 
Speit, Andreas. 2017. Reichsbürger - Eine facettenreiche, gefährliche Bewegung. In Reichsbürger. Die unterschätzte Gefahr, Hrsg. Andreas Speit, 9-21. Berlin: Ch. Links.

Stöss, Richard. 2010. Rechtsextremismus im Wandel. Bonn: Bonner Universitätsdruckerei.

Ullrich, Carsten G. 2020. Das diskursive Interview. Wiesbaden: Springer VS.

Volkov, Shulamit. 2000. Antisemitismus als kultureller Code. Zehn Essays. München: Beck.

Vormbaum, Moritz. 2017. „Reichsbürger“ und Strafrecht. Juristische Rundschau 10:503-512.

Weber, Max. 2002. Wirtschaft und Gesellschaft. Grundrisse der verstehenden Soziologie. Neu-Isenburg: Melzer.

Weiß, Volker. 2017. Die autoritäre Revolte. Die Neue Rechte und der Untergang des Abendlandes. Stuttgart: Klett-Cotta.

Wiedemann, Felix. 2016. Das Verhältnis der extremen Rechten zur Religion. In Handbuch Rechtsextremismus, Hrsg. Fabian Virchow, Martin Langebach, und Alexander Häusler, 511-532. Wiesbaden: Springer VS.

Witzel, A. 2000. Das problemzentrierte Interview. http://www.qualitative-research.net/index.php/fqs/ article/viewArticle/1132/2519. Zugegriffen: 8. Nov. 2020.

Wood, Michael J., Karren M. Douglas, und Robbie M. Sutton. 2012. Dead and alive: beliefs in contradictory conspiracy theories. Social Psychological and Personality Science 3:767-773.

Wössner, Jakobus. 1973. Systemanalyse und „Religiöse Bedürfnisse“. In Zur Theorie der Religion. Religion und Sprache, Hrsg. Günter Dux, Thomas Luckmann, und Joachim Matthes, 133-144. Wiesbaden: Springer.

Zeit Online. 2016a. „Reichsbürger“ verletzt Polizisten lebensgefährlich. https://www.zeit.de/gesellschaft/ zeitgeschehen/2016-10/georgensgmuend-razzia-wohnungsdurchsuchung-schuesse-polizisten. Zugegriffen: 15. Dez. 2019.

Zeit Online. 2016b. Opposition wirft Verfassungsschutz Versagen vor. https://www.zeit.de/politik/ deutschland/2016-10/reichsbuerger-verfassungsschutz-ueberwachung-die-gruene-vorwuerfe/komp lettansicht. Zugegriffen: 6. Juli 2020.

\section{Quellen}

Amt für Menschenrechte. 2019a. Fragen und Antworten. http://www.ichr.de/fragen-und-antworten.html. Zugegriffen: 29. Nov. 2019.

Amt für Menschenrechte. 2019b. GdM Ziel und Arbeitsweise. http://www.ichr.de/gdm-ziel-und-arbeits weise.html. Zugegriffen: 29. Nov. 2019.

Deutsches Kolleg. 2019a. Zerlegung der Lage. http://brd-ende.com/1992/11/zerlegung-der-lage/. Zugegriffen: 14. Dez. 2019.

Deutsches Kolleg. 2019b. Dutschke und Hitler. http://brd-ende.com/schublade/erklaerungen/. Zugegriffen: 14. Dez. 2019.

Deutsches Kolleg. 2019c. Das Gesetz des Nomadentums. http://brd-ende.com/schublade/viertes-reich/ schulungsschriften/lehre-vom-gemeinwesen/. Zugegriffen: 15. Dez. 2019.

Europäische Aktion. 2019a. „Auf das Leben“ - Traditionelle Osterfeier. https://europaeische-aktion.org/ 2017/04/16/auf-das-leben-traditionelle-osterfeier/?v=3a52f3c22ed6. Zugegriffen: 16. Dez. 2019.

Europäische Aktion. 2019b. Ideologie und Weltanschauung - Eine Gegenüberstellung. https://europaeischeaktion.org/2017/07/20/ideologie-und-weltanschauung-eine-gegenueberstellung/?v=3a52f3c22ed6. Zugegriffen: 16. Dez. 2019.

Europäische Aktion. 2019c. Europas Totenglocken läuten - Visuelle Intervention in Sangerhausen. https://europaeische-aktion.org/2016/12/21/europas-totenglocken-laeuten-visuelle-intervention-insangerhausen/?v=3a52f3c22ed6. Zugegriffen: 17. Dez. 2019.

Exil-Regierung Deutsches Reich. 2019a. Die althochdeutsche Sprache - Teil 1. Die Deutsche Sprache trägt in sich die Göttlichkeit, das Göttliche, den Ur-Gott. http://friedensvertrag.org/index.php/lexikon-2/ 59-die-althochdeutsche-sprache-teil-1. Zugegriffen: 8. Dez. 2019.

Staatenlos.info. 2019a. Bevölkerungsreduzierung und Eugenik. https://staatenlos.info/ursache-von-derheimat-in-die-staatenlosigkeit/neue-weltordnung-der-faschisten-5-reich/bevoelkerungsreduzierungund-eugenik. Zugegriffen: 20. Dez. 2019.

Staatenlos.info. 2019b. Ideologien und Religionen. https://staatenlos.info/themen-und-sachgebiete/ ideologien-religionen. Zugegriffen: 20. Dez. 2019.

Staatenlos.info. 2019c. Geplant organisierter Bevölkerungsaustausch (ethnische Säuberung) mit Aufbau eines „Islamischen Staates - Kalifat“ durch den „Tiefen Staat - Deep Staate“ in Deutschland und Eu- 
ropa? https://staatenlos.info/projekt-islamischer-staat-in-deutschland-europa/geplant-organisierterbevoelkerungsaustausch. Zugegriffen: 20. Dez. 2019.

Niklas Herrberg Wissenschaftlicher Mitarbeiter am Lehrstuhl für Soziologie mit den Schwerpunkten Politische Kulturen sozialer Ungleichheit und empirische Sozialforschung (Prof. Dr. Heiko Beyer) 